\title{
Simultaneous ram pressure and tidal stripping How dwarf spheroidals lost their gas
}

\author{
Journal Article \\ Author(s): \\ Mayer, Lucio; Mastropietro, Chiara; Wadsley, James; Stadel, Joachim; Moore, Ben \\ Publication date: \\ 2006-07 \\ Permanent link: \\ https://doi.org/10.3929/ethz-b-000024095 \\ Rights / license: \\ In Copyright - Non-Commercial Use Permitted \\ Originally published in: \\ Monthly Notices of the Royal Astronomical Society 369(3), https://doi.org/10.1111/j.1365-2966.2006.10403.x
}




\title{
Simultaneous ram pressure and tidal stripping; how dwarf spheroidals lost their gas
}

\author{
Lucio Mayer, ${ }^{1,2 \star}$ Chiara Mastropietro, ${ }^{2}$ James Wadsley, ${ }^{3}$ Joachim Stadel $^{2}$ \\ and Ben Moore ${ }^{2}$ \\ ${ }_{1}^{1}$ Institut für Astronomie, Physics Department, ETH Zurich, CH-8093 Zurich, Switzerland \\ ${ }^{2}$ Institute of Theoretical Physics, University of Zürich, Winterthurerstrasse 190, 8057 Zurich, Switzerland \\ ${ }^{3}$ Department of Physics \& Astronomy, McMaster University, 1280 Main St. West, Hamilton, ON L8S 4M1, Canada
}

Accepted 2006 March 21. Received 2006 January 19; in original form 2005 April 14

\begin{abstract}
We perform high-resolution $N$-body + SPH (smoothed particle hydrodynamics) simulations of gas-rich dwarf galaxy satellites orbiting within a Milky Way-sized halo and study for the first time the combined effects of tides and ram pressure. The structure of the galaxy models and the orbital configurations are chosen in accordance with those expected in a Lambda cold dark matter $(\Lambda \mathrm{CDM})$ universe. While tidal stirring of disky dwarfs produces objects whose stellar structure and kinematics resembles that of dwarf spheroidals after a few orbits, ram pressure stripping is needed to entirely remove their gas component. Gravitational tides can aid ram pressure stripping by diminishing the overall potential of the dwarf, but tides also induce bar formation which funnels gas inwards making subsequent stripping more difficult. This inflow is particularly effective when the gas can cool radiatively. Assuming a low density of the hot Galactic corona consistent with observational constraints, dwarfs with $V_{\text {peak }}<$ $30 \mathrm{~km} \mathrm{~s}^{-1}$ can be completely stripped of their gas content on orbits with pericenters of $50 \mathrm{kpc}$ or less. Instead, dwarfs with more massive dark haloes and $V_{\text {peak }}>30 \mathrm{~km} \mathrm{~s}^{-1}$ lose most or all of their gas content only if a heating source keeps the gas extended, partially counteracting the bar-driven inflow. We show that the ionizing radiation from the cosmic ultraviolet (UV) background at $z>2$ can provide the required heating. In these objects, most of the gas is removed or becomes ionized at the first pericenter passage, explaining the early truncation of the star formation observed in Draco and Ursa Minor. Galaxies on orbits with larger pericenters and/or falling into the Milky Way halo at lower redshift can retain significant amounts of the centrally concentrated gas. These dwarfs would continue to form stars over a longer period of time, especially close to pericenter passages, as observed in Fornax and other dwarf spheroidal galaxies (dSphs) of the Local Group. The stripped gas breaks up into individual clouds pressure confined by the outer gaseous medium that have masses, sizes and densities comparable to the $\mathrm{H}$ I clouds recently discovered around M31.
\end{abstract}

Key words: hydrodynamics - methods: $N$-body simulations - galaxies: dwarf - galaxies: interactions - Local Group.

\section{INTRODUCTION}

The origin of dwarf spheroidal galaxies (dSphs) is a long standing subject of debate. These galaxies are gas poor or completely devoid of gas and are the faintest galaxies known. Objects matching the definition of dSphs are found both in galaxy clusters and in groups, but it is certainly the Local Group the place where dSphs have been better studied thanks to their proximity.

^E-mail: lucio@phys.ethz.ch
Two main formation paths have been proposed to explain their origin, nature of nurture. In one scenario, these faint galaxies could be the result of cosmic reionization and stellar feedback quenching gas accretion and star formation in low-mass haloes (Dekel \& Silk 1986; Thoul \& Weinberg 1996; Bullock, Kravtsov \& Weinberg 2000; Ferrara \& Tolstoy 2000; Benson et al. 2002a,b; Somerville 2002; Susa \& Umemura 2004). However, there is no obvious signature of reionization in the star formation history of these galaxies (Grebel \& Gallagher 2004), which in many cases continued to form stars over more than $10 \mathrm{Gyr}$, and for some $\mathrm{dSphs}$ the most recent inferred halo masses are likely too large $\left(10^{8}-10^{9} \mathrm{M}_{\odot}\right.$; see 
Kleyna et al. 2002; Kazantzidis et al. 2004; Wilkinson et al. 2004) for supernovae winds to remove most of the baryons (Mori, Ferrara \& Madau 2002; Mayer \& Moore 2004; Read \& Gilmore 2004).

In addition, these models do not naturally explain why dSphs share some structural similarities with equally faint but gas-rich, much younger disc-like galaxies, dwarf irregulars (dIrrs), notably their exponential light distribution. Furthermore, they do not give rise to the morphology-density relation observed in groups and clusters, namely the fact that $\mathrm{dSphs}$ tend to be concentrated towards the central galaxies while dIrrs are found at much larger distances, nor they explain the dichotomy between dominance of rotation for the stars of most dIrrs and dominance of pressure support for those of dSphs. Various environmental effects have been scrutinized to explain similarities and differences between the two types of galaxies postulating that dSphs are somehow transformed dIrrs. Ram pressure stripping of gas from dIrr-like progenitors caused by a hot diffuse gaseous corona surrounding the Milky Way has been proposed as a way to explain the low gas contents of $\mathrm{dSphs}$ together with the similar stellar light distributions of the two types of dwarfs (Einasto et al. 1974; Faber \& Lin 1983; Grebel, Gallagher \& Herbeck 2003), and has also been considered as the origin of $\mathrm{H}$ I clouds possibly associated with some dSphs and transitional dIrrs/dSphs-like Phoenix (Blitz \& Robishaw 2000; Gallart et al. 2001). Van den Bergh (1996) has argued that the correlation between galactocentric distance and gas content in $\mathrm{dSphs}$ is a signature of ram pressure stripping. Tidal stripping of dIrrs has also been considered as an alternative way of removing gas from the dwarfs (e.g. Ferguson \& Binggeli 1994).

Recently, high-resolution $N$-body/SPH (smoothed particle hydrodynamics) simulations were used to show that tides do not only remove gas and stars but can also reshape the stellar components of a dwarf disky galaxy resembling a dIrr (Mayer et al. 2001a,b). This 'tidal stirring' can turn a rotationally supported disc into a pressuresupported spheroidal stellar system by means of bar formation and a subsequent buckling instability. The mechanism is particularly effective for satellites of massive galaxies that are strongly tidally shocked owing to the plunging orbits expected in cold dark matter (CDM) models and works for systems having a variety of dark halo profiles, including cuspy profiles (Mayer et al. 2002). The transformation is not necessarily associated with intense tidal mass loss; indeed, objects with very high mass-to-light ratios (M/L), thus embedded in dense, massive dark haloes, can still be reshaped from the instabilities induced by tidal shocks albeit losing minimal mass (Mayer et al. 2001b).

A problem of this model, however, is that tides cannot remove completely the gas from the disky progenitors of dSphs; the low gas fractions of dSphs can be explained by only invoking rapid consumption from star formation of the remaining gas in a series of tidally triggered bursts associated with bar-driven gas inflows (Mayer et al. 2001b). However, although intermittent star formation is observed in some dSphs (Gallart et al. 1999; Hernandez, Gilmore \& Valls-Gabaud 2000), the star formation rates needed to completely consume the gas are well in excess of those implied by reconstructions of the star formation histories of dSphs using detailed colour-magnitude diagrams (Mayer et al. 2001b). Moreover, Draco and a few other dSphs do not show extended star formation histories, instead seem to have ceased star formation about $10-\mathrm{Gyr}$ ago (Grebel \& Gallagher 2004).

It is likely that both gravitational tides and hydrodynamical processes play a role (Gavazzi et al. 2001). For example, ram pressure could act simultaneously with tides and increase the efficiency of gas removal (Mayer \& Wadsley 2004). The effect of ram pressure on the gas content of spiral galaxies has been widely stud- ied for galaxies in clusters in a number of numerical works since the early 80s, among them Farouki \& Shapiro (1980), Toyama \& Ikeuchi (1980), Vollmer et al. (1999, 2000, 2001), Schulz \& Struck (2001), Abadi, Moore \& Bower (1999), Quilis \& Moore (2001) and Vollmer (2003). Both particle-based methods, such as SPH, and grid-based methods have been used in such works. More recently, Roediger \& Hensler (2005) have studied ram pressure stripping of spiral galaxies in lower density environments, their models being applicable to both poor groups and the outskirts of galaxy clusters. They found that, although stripping is much less severe than in the cores of clusters, the compression exerted by the outer medium is still capable of altering significantly the distribution of the gas component and some mass loss can still occur. This suggests that the effect on galaxies with much shallower potential wells such as dwarf galaxies might be quite dramatic even in such low-density environments. Recent 2D and 3D grid-based hydrodynamical simulations of ram pressure stripping of disc-like dwarfs in poor groups show that ram pressure alone can remove most of the gas in dwarf galaxies with halo circular velocities lower than $30 \mathrm{~km} \mathrm{~s}^{-1}$ (Marcolini, Brighenti \& D'Ercole 2003, 2004). However, these and other simulations (Mori \& Burkert 2000) do not use models of dwarf galaxies directly based on the Lambda cold dark matter $(\Lambda \mathrm{CDM})$ model and do not include the effect of tides. The effectiveness of both ram pressure and tidal stirring will also depend on the orbital history of the dwarfs, and this is where the cosmological framework comes into play. For example, dwarfs that fell into the primary halo at very high redshift likely had orbits with smaller pericenters relative to those falling later on (Mayer et al. 2001b), thus suffering stronger tidal shocks, and possibly also stronger ram pressure in a denser gaseous corona, while being exposed to the effect of the cosmic ultraviolet (UV) background (Barkana \& Loeb 1999; Bullock et al. 2000; Somerville 2002; Shaviv \& Dekel 2003). Indeed, cosmological simulations of galaxy formation incorporate all such mechanisms, but their resolution is still too coarse to allow a study of their effects at the scale of the tiny dwarf spheroidals (Governato et al. 2004).

In this paper, we will study for the first time the combined effects of ram pressure and tidal stirring using high-resolution threedimensional $N$-body+SPH simulations of dwarf galaxies orbiting within the Milky Way dark halo and gaseous corona. We will use galaxy models and orbits consistent with the predictions of $\Lambda \mathrm{CDM}$ simulations. The simulations include also radiative cooling as well as heating and ionization from the cosmic UV background radiation. The main goal of the present work will be to establish whether these two mechanisms can explain the present-day low gas content of dSphs if these galaxies are the descendants of gas-rich dwarfs with structure similar to today's dIrrs.

\section{INITIAL CONDITIONS}

\subsection{Galaxy models}

Models comprise an exponential disc of gas and stars embedded in a live Navarro-Frenk-White (NFW) halo for the dwarf (Navarro, Frenk \& White 1996), and a live NFW halo plus hot gas distribution with the same density profile for the primary galaxy. Halo virial parameters (mass, radius and circular velocity) are consistent with the $\Lambda \mathrm{CDM}$ model. Although we believe that the Milky Way halo has a virial velocity $V_{\text {vir }}=140-160 \mathrm{~km} \mathrm{~s}^{-1}$ (Klypin, Zhao \& Somerville 2002), we use a slightly larger value, $V_{\text {vir }}=180 \mathrm{~km} \mathrm{~s}^{-1}$, in order to have $V_{\text {peak }}=230 \mathrm{~km} \mathrm{~s}^{-1}$ at about $10 \mathrm{kpc}$ from the centre without including a disc component. We choose a concentration $c=10$ for the Milky Way (MW) halo, this being typical for $\Lambda \mathrm{CDM}$ haloes 
at a scale of $\sim 10^{12} \mathrm{M}_{\odot}$ (Bullock et al. 2001). With our choice of parameters, the shape of the rotation curve is quite flat out to $100 \mathrm{kpc}$ and the resulting halo mass within $100 \mathrm{kpc}$ is $\sim 8 \times 10^{11} \mathrm{M}_{\odot}$, consistent with the orbital dynamics of the Magellanic Clouds and distant dSphs (Lin, Jones \& Klemola 1995; Dehnen \& Binney 1998). Overall our halo model resembles model A3 in Klypin et al. (2002), which matches simultaneously most of the known observational constraints within the framework of the $\Lambda \mathrm{CDM}$ model. The hot gas is in hydrostatic equilibrium within the halo potential and its temperature is $\sim 10^{6} \mathrm{~K}$ at $50 \mathrm{kpc}$, consistent with $\mathrm{O}$ VI, O VII and $\mathrm{X}$-ray absorption measurements in the Galactic halo (Sembach et al. 2003). Assuming an isotropic model, the halo temperature at a given radius $r$ is determined by the cumulative mass distribution $M(r)$ of the dark, stellar and gaseous components of the MW beyond $r$ and by the density profile $\rho_{\mathrm{h}}(r)$ of the hot gas:

$T(r)=\frac{m_{\mathrm{p}}}{k_{\mathrm{B}}} \frac{1}{\rho_{\mathrm{h}}(r)} \int_{r}^{\infty} \rho_{\mathrm{h}}(r) \frac{G M(r)}{r^{2}} \mathrm{~d} r$,

where $m_{\mathrm{p}}$ is the proton mass, $G$ and $k_{\mathrm{B}}$ are the gravitational and Boltzmann constants, respectively. Its density at $50 \mathrm{kpc}$ from the centre is $8 \times 10^{-5}$ atoms cm$~^{-3}$ (see also Mastropietro et al. 2005). At a distance of $\sim 100 \mathrm{kpc}$ or more, where the dwarf galaxies that we have simulated spend most of the time while orbiting within the primary halo (see below), the hot halo density is $<10^{-5}$ atoms $\mathrm{cm}^{-3}$. Such a hot diffuse halo is also a natural expectation of the current $\Lambda$ CDM paradigm of structure formation (White \& Frenk 1991; Sommer-Larsen, Gotz \& Portinari 2003; Governato et al. 2004; Maller \& Bullock 2004).

The multicomponent dwarf galaxy models are built using the technique developed by Hernquist (1993) and later refined by Springel \& White (1999) (for further details on the modelling, see also Mayer et al. 2002). They comprise an exponential disc of gas and stars embedded in an NFW halo. We used three dwarf models with $V_{\text {peak }}=$ 28,42 or $62 \mathrm{~km} \mathrm{~s}^{-1}$ and concentration $c=4$ (the first two mod-
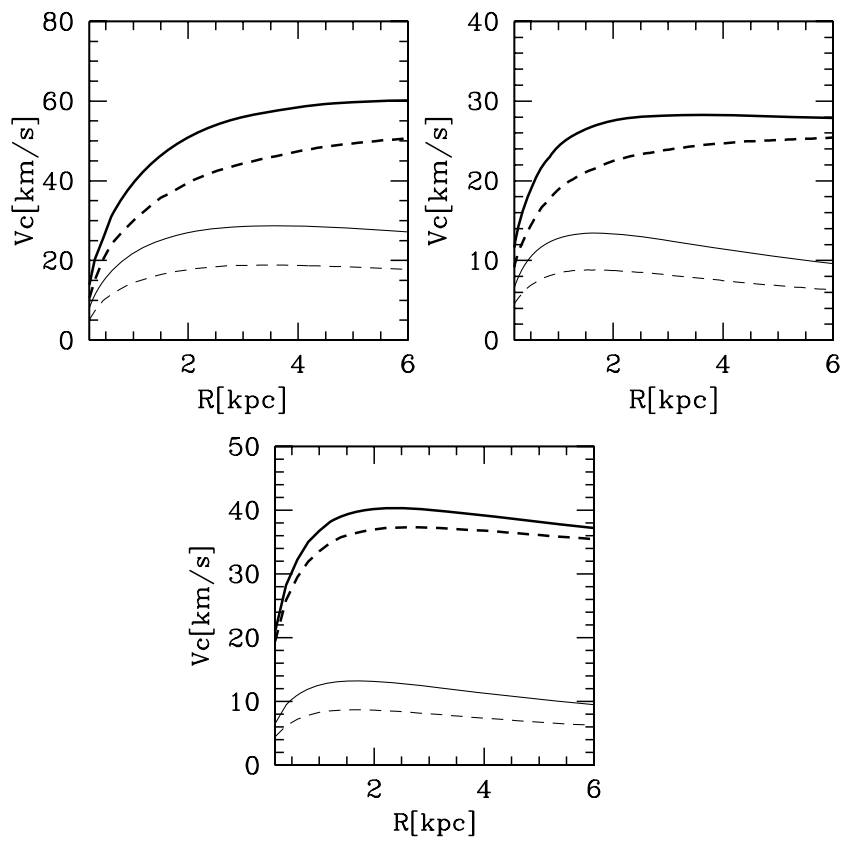

Figure 1. Initial rotation curves of the dwarf galaxy models. The lines show, respectively, the total rotation curve (thick solid line), the contribution of dark matter (thick dashed line), that of stars (thin solid line) and that of gas (thin dashed line). From top left to bottom, models V60c4, V30c4 and V40c20 are shown. els) and $c=20$ (the third model). The choice of halo concentration can be quite flexible since cosmological simulations show a large scatter of such property at small mass scales, mostly due to a large spread in halo formation times (Bullock et al. 2001). The rotation curves of the models are reported in Fig. 1, while the rotation curves of models V60c4 and V30c4 are slowly rising in agreement with those of today's dIrrs, that of model V40c20 rises quite steeply owing to the much higher halo concentration. A steep rotation curve is a more appropriate choice for the progenitors of Draco or Ursa Minor that have very high central dark matter densities (see Hogan \& Dalcanton 2000; Mayer et al. 2001b).

Lokas (2002) and Kazantzidis et al. (2004) have shown that dSphs kinematics suggests that they are embedded in haloes with $V_{\text {peak }} \sim$ $20-35 \mathrm{~km} \mathrm{~s}^{-1}$. We used even larger values of $V_{\text {peak }}$ in our initial conditions since these can later decrease as a result of tidal mass loss, by roughly a factor of 2 according to the recent cosmological simulations by Kravtsov, Gnedin \& Klypin (2004).

The galaxy models have discs of mass $\approx 4$ per cent of the total mass, lower than the universal baryon fraction but quite typical for present-day low surface brightness (LSB) or dIrr galaxies (Jimenez, Verde \& Oh 2003). Following Mo, Mao \& White (1998), the exponential scalelength $R_{\mathrm{h}}$ of the disc is determined by the spin parameter and concentration $c$ of the halo; we use $\lambda=0.043$ for models Rc4a and Rc4b, and $\lambda=0.08$ for model Rc20a,b (the mean spin of cosmological haloes is $\lambda \sim 0.05$, see e.g. Gardner 2001 , and 20 per cent of haloes have spins as high as 0.1 due to the lognormal shape of the probability distribution of halo spins). The disc sizes in models $\mathrm{V} 28 \mathrm{c} 4$ and V40c20 are the same (the higher spin parameter compensates for the higher halo concentration) and, since also the disc mass is the same by choice, we can isolate the role of halo concentration (or, equivalently, $V_{\text {peak }}$ ) by comparing the two models. Note that all the models have a central surface brightness of 23 mag $\operatorname{arcsec}^{-2}$ in the $B$ band for a stellar M/L of 2, typical of dIrrs and dwarf spirals (see de Blok \& McGaugh 1997). Galaxies with these low disc mass fractions are stable to bar formation in isolation irrespective of halo concentration (Mayer \& Wadsley 2004b). However, they can become bar unstable when tidally perturbed by a much more massive halo.

We use $3 \times 10^{5}$ particles for the dark haloes of the dwarfs and $3 \times 10^{4}$ particles for both the stellar and the gaseous disc of the dwarf. The Milky Way halo is modelled with $10^{6}$ dark matter particles, while $5 \times 10^{5}$ particles are used for the superimposed gaseous halo (the mass of hot halo particles is $8 \times 10^{4} \mathrm{M}_{\odot}$ ). Each simulation has a total of $\sim 1.8 \times 10^{6}$ particles, which makes them quite computationally demanding. Two runs employing model V60c4 were repeated with increased resolution in the disc of the dwarf, $\left(2 \times 10^{5}\right.$ particles for both its stellar and its gaseous component, see Table 1). The choice of putting a large fraction of the total number of particles in the primary halo is motivated by the desire of minimizing spurious two-body heating between halo particles and those of the discs of the dwarfs (Moore, Katz \& Lake 1996; Mayer 2004). We use a fairly large gravitational softening for the gas and dark matter particles in the primary system, $\varepsilon=2 \mathrm{kpc}$, to minimize discreteness noise in the potential. For the dwarf models instead, the softening is $\varepsilon=0.3 R_{\mathrm{h}}$ for halo particles and $\varepsilon=0.1 R_{\mathrm{h}}$ for star and gas particles, where $R_{\mathrm{h}}$ is the disc scalelength (see Table 1 ).

\subsection{Simulations}

The dwarf galaxy satellites are placed on bound eccentric orbits in the primary halo. In Mayer et al. (2001b), we explored a wide range of orbital eccentricities and initial disc inclinations. Tidally 
Table 1. Parameters of the FI simulations. Column 1: name of model; Column 2: peak circular velocity of the satellite $\left(\mathrm{km} \mathrm{s}^{-1}\right)$; Column 3: satellite halo concentration; Column 4: disc scalelength (kpc); Column 5: apocenter distance (kpc); Column 6: pericenter distance (kpc); Column 7: thermal physics $(\mathrm{AD}=$ adiabatic, $\mathrm{RC}=$ radiative cooling, $\mathrm{RCUV}=$ radiative cooling + cosmic UV background). All runs include tides and ram pressure except those referred to as TD which do not include ram pressure. Column 8: name of run (HR stands for high resolution, see the text). Column 9: initial gas mass (in units of $10^{6} \mathrm{M}_{\odot}$ ); Column 10: final gas mass (in units of $10^{6} \mathrm{M}_{\odot}$ ).

\begin{tabular}{lccccccccc}
\hline Model & $V_{\text {peak }}$ & $c$ & $R_{\mathrm{h}}$ & $r_{\text {apo }}$ & $r_{\text {peri }}$ & Physics & Run & $M_{\text {gas }_{\mathrm{i}}}$ & $M_{\text {gas }_{\mathrm{f}}}$ \\
\hline V60c4 & 62 & 4 & 2.2 & 250 & 50 & AD & Rc4aAD & 450 & 55 \\
V60c4 & 62 & 4 & 2.2 & 250 & 50 & AD & Rc4aADHR & 450 & 60 \\
V60c4 & 62 & 4 & 2.2 & 250 & 50 & RC & Rc4aRCHR & 450 & 380 \\
V60c4 & 62 & 4 & 2.2 & 250 & 50 & RCUV & Rc4aRCUV & 450 & 290 \\
V28c4 & 25 & 4 & 1.1 & 250 & 50 & TD(AD) & Rc4bTD & 45 & 20 \\
V28c4 & 25 & 4 & 1.1 & 250 & 50 & AD & Rc4bAD & 45 & 0 \\
V28c4 & 25 & 4 & 1.1 & 250 & 50 & RC & Rc4bRC & 45 & 9.5 \\
V28c4 & 25 & 4 & 1.1 & 150 & 30 & RC & Rc4cRC & 45 & 0 \\
V40c20 & 42 & 20 & 0.5 & 150 & 30 & TD(AD) & Rc20TD & 46.5 & 22.2 \\
V40c20 & 42 & 20 & 0.5 & 150 & 30 & AD & Rc20AD & 46.5 & 0 \\
V40c20 & 42 & 20 & 0.5 & 150 & 30 & RC & Rc20RC & 46.5 & 3.55 \\
V40c20 & 42 & 20 & 0.5 & 150 & 30 & RCUV & Rc20RCUV & 46.5 & 0.65 \\
V40c20 & 42 & 20 & 0.5 & 250 & 50 & AD & Rc20bAD & 46.5 & 0 \\
V40c20 & 42 & 20 & 0.5 & 250 & 50 & RC & Rc20bRC & 46.5 & 10 \\
\hline
\end{tabular}

induced transformation was found to be essentially independent of disc inclination (with only purely retrograde encounters versus purely prograde encounters showing significant differences). We also found that the orbital time, and not the orbital eccentricity, is the most sensitive orbital parameter for causing the transformation since it determines both the strength and number of tidal shocks. Here, we choose orbits with an apocenter-to-pericenter ratio of 5, corresponding to the mean value for satellites in cosmological simulations (Ghigna et al. 1998; Gill, Knebe \& Gibson 2004), and we consider apocenters of 250 or $150 \mathrm{kpc}$ (the pericenter being 50 or $30 \mathrm{kpc}$ ) corresponding to orbital times of, respectively, 3.2 and $1.3 \mathrm{Gyr}$. The dwarf galaxies start at apocenter and are evolved for three to five orbits with their discs initially inclined $60^{\circ}$ with respect to their orbital plane. Different mean orbital distances radii could be associated with satellites infalling into the Milky Way halo at different cosmic epochs (Mayer et al. 2001b; Gill et al. 2004; Kravtsov et al. 2004), say at $z>1$ or $z<1$, for, respectively, the smaller and larger orbital time.

The simulations were performed with the parallel Tree $+\mathrm{SPH}$ code GASOLINE (Wadsley, Stadel \& Quinn 2004). The internal energy of the gas is integrated using the asymmetric formulation, that gives results very close to the entropy conserving formulation (Springel \& Hernquist 2003) but conserves energy better. In simulations without radiative cooling, the gas can heat or cool only by adiabatic compression or expansion. Dissipation in shocks is modelled using the quadratic term of the standard Monaghan artificial viscosity. The Balsara correction term is used to reduce unwanted shear viscosity (Balsara 1995). A detailed description of the SPH code is given in Wadsley et al. (2004).

We perform both adiabatic runs and runs with radiative cooling. We use a standard cooling function for a primordial mixture of hydrogen and helium (the metallicity in dSphs is indeed much lower than solar, with $-1<\langle\mathrm{Fe} / \mathrm{H}\rangle<-2$, see Gallagher et al. 2003). Cooling shuts off below $10^{4} \mathrm{~K}$. The initial temperature of the gaseous discs is $8000 \mathrm{~K}$ for the most massive model $\left(V_{\max }=62 \mathrm{~km} \mathrm{~s}^{-1}\right)$ and slightly lower for the other lighter models. The purpose of running adiabatic simulations is two-fold; first, it helps in understanding the interplay between thermodynamical and mechanical effects of ram pressure as we shall see in the following section, and second heating by external radiation fields or stellar feedback, especially in low-mass objects as those considered in this paper, might overtake radiative cooling and create a hot phase that would essentially behave as an adiabatic gas (Thacker \& Couchman 2000; Marcolini et al. 2003; Springel \& Hernquist 2003). Therefore, radiative cooling and adiabatic runs can be regarded as two extreme cases crudely bracketing the conditions of the real interstellar medium (ISM) in which a hot and a cold phase will coexist at all times. Finally, in a few runs with cooling we also include a uniform external radiation field which models the cosmic UV background arising at high redshift. This is implemented using the Haardt \& Madau (1996) model which includes photoionizing and photoheating rates produced by quasi-stellar objects and galaxies starting at $z=7$. We implicitly assume that the dwarf enters the Milky Way halo at $z=7$. The ionizing flux remains quite high until $z=2$, therefore the dwarf is exposed to the radiation for a duration of $\sim 5 \mathrm{Gyr}$.

We complement our 'full interaction' runs (hereafter FI runs), so called because they include both tides and ram pressure, with wind tunnel (WT) simulations aimed at studying the effect of ram pressure alone. The volume of the simulations is a tube with rectangular section, having a base equal to the diameter of the dwarf model (out to its virial radius) and height $h=v t$, where $v$ is the typical velocity of the satellite at pericenter in the FI runs $\left(290 \mathrm{~km} \mathrm{~s}^{-1}\right)$ and $t$ is set $\sim 0.5 \mathrm{Gyr}$ ( $\gtrsim t_{\text {enc }}=R_{\text {peri }} / V_{\text {peri }}$, where $t_{\text {enc }}$ is the time the dwarf spends near pericenter of the orbits in the FI runs). We represent the hot gas as a flux of particles moving with a velocity $v$ along the longest axis of the tube, this being parallel or at an angle with the angular momentum of the disc depending on the run. The hot particles have an initial random distribution, a temperature $T=$ $10^{6} \mathrm{~K}$ and a number density $n_{\mathrm{h}}=8 \times 10^{-5} \mathrm{~cm}^{-3}$. We use periodic boundary conditions in order to restore the flow of hot gas that leaves the tube. We will indicate these as WT runs (where WT stands for 'wind tunnel'). These experiments allow to resolve the background gas flow with very high resolution, thus limiting numerical artefacts like the spurious stripping induced by transfer of momentum in twobody collisions between the hot halo particles and the gas particles in the disc of the dwarf (Abadi et al. 1999). The mass of the gas particles is equal to that used for the dwarfs in the FI runs, i.e. $8 \times 10^{4} \mathrm{M}_{\odot}$, for the runs employing our largest dwarf model, V60c4, while it is eight times lower for the runs employing the other two models, V28c4 and V40c20, since these have correspondingly lighter discs. The total number of particles used in the hi-resolution WT runs thus varies from $10^{6}$ in runs with models V28c4 and V40c20 to $2 \times 10^{5}$ in runs with model V60c4. The (adiabatic) runs with models $\mathrm{V} 28 \mathrm{c} 4$ and V40c20 where repeated with a lower resolution of the background flow, equal to that of the FI runs. In addition, run T2c20 (which includes radiative cooling) was repeated at three times higher resolution than that of the original run (run T2c20HR in Table 2), for a total of more than $3 \times 10^{6} \mathrm{SPH}$ particles.

\section{RESULTS}

Tables 1 and 2 report the results of the FI and WT runs. Dwarf galaxies undergo severe stripping owing to the combined action of tides and ram pressure. The overall mass loss varies depending on the dwarf galaxy model, in particular the depth of its potential well, on its orbit and on the gas physics (the balance between cooling and heating). Dwarfs can retain 20-50 per cent of their initial gas content or be completely stripped. Ram pressure stripping occurs 
Table 2. Parameters of the WT simulations. Column 1: model galaxy (see Table 1). Column 2: gas physics ( $\mathrm{AD}=$ adiabatic, $\mathrm{RC}=$ radiative cooling). Column 3: angle between $z$-axis of the disc and direction of the flow. Column 4: mass remaining in the disc at after $5 \times 10^{8} \mathrm{yr}$ (in units of $10^{7} \mathrm{M}_{\odot}$ ). Column 5: stripping radius in $\mathrm{kpc}$. Column 6: stripping radius (in $\mathrm{kpc}$ ) predicted using analytical prediction (see the text). Column 7: name of run (LR stands for 'low resolution', see the text).

\begin{tabular}{lcccccc}
\hline Model & Gas physics & $i$ & $M_{\text {fin }}$ & $R_{\text {strip }_{1}}$ & $R_{\text {strip }_{2}}$ & Run \\
\hline V28c4 & AD & 0 & 0 & 0 & 0 & T1c4a \\
V28c4 & AD & 90 & 0 & 0 & 0 & T2c4a \\
V28c4 & RC & 0 & 0 & 0 & 0 & T3c4a \\
V28c4 & RC & 45 & 0 & 0 & 0 & T4c4a \\
V28c4 & RC & 90 & 6 & 0 & 0 & T5c4a \\
V60c4 & AD & 0 & 57 & 4.25 & 5.8 & T1c4b \\
V60c4 & AD & 30 & 66 & 4.25 & 5.8 & T2c4b \\
V60c4 & AD & 60 & 64 & 4.25 & 5.8 & T3c4b \\
V40c20 & AD & 0 & 4.38 & 1.58 & 1.6 & T1c20 \\
V40c20 & AD & 0 & 3.6 & 1.5 & 1.6 & T1c20LR \\
V40c20 & RC & 0 & 6.2 & 1.75 & 1.6 & T2c20 \\
V40c20 & RC & 0 & 6.85 & 2 & 1.6 & T2c20HR \\
V40c20 & RC & 90 & 7.85 & 2 & 1.6 & T3c20 \\
\hline
\end{tabular}

very rapidly as the galaxy approaches pericenter for the first time. A bow shock is visible (Fig. 2) and reflects the fact that the dwarfs near pericenter are moving at mildly supersonic velocities in the hot halo owing to their eccentric orbits (the Mach number is $\sim 1.5$ ).
The tidal shock at first pericenter passage deforms the disc to an extent that depends on how deep is the potential well of the galaxy. Symmetric stellar tidal tails develop but the gas generally trails the galaxy since ram pressure sweeping dominates (compare gas and stars in Fig. 3). The dark halo loses most of its mass in all cases and the overall depth of the potential well is reduced which increases the efficiency of gas removal. The stars develop a bar instability due to the strong tidal perturbation (Fig. 3) which drives a radial gas inflow (Mayer et al. 2001b). This inflow counteracts the reduction of the mean restoring force by moving some gas deeper towards the centre of the dwarf where it is harder to strip (note the central density concentration in Figs 4-6). As a result, on subsequent orbits ram pressure stripping continues but with much lower intensity. In what follows, we will describe the results quantitatively by analysing the gas mass loss in FI and WT runs as well as the fate of the stripped gas.

\subsection{Gas mass loss}

\subsubsection{WT runs}

Figs 7-10 show the evolution of the gas component of the dwarfs. In the absence of an external perturbation, the stellar discs are stable to bar formation. Removal of gas begins immediately; a bow shock develops and some gas in the disc rises above the disc plane slightly more than $10^{7} \mathrm{yr}$. This is evident in the early truncation of the gas surface density profiles (see Fig. 11). The stripped gas is diffuse in

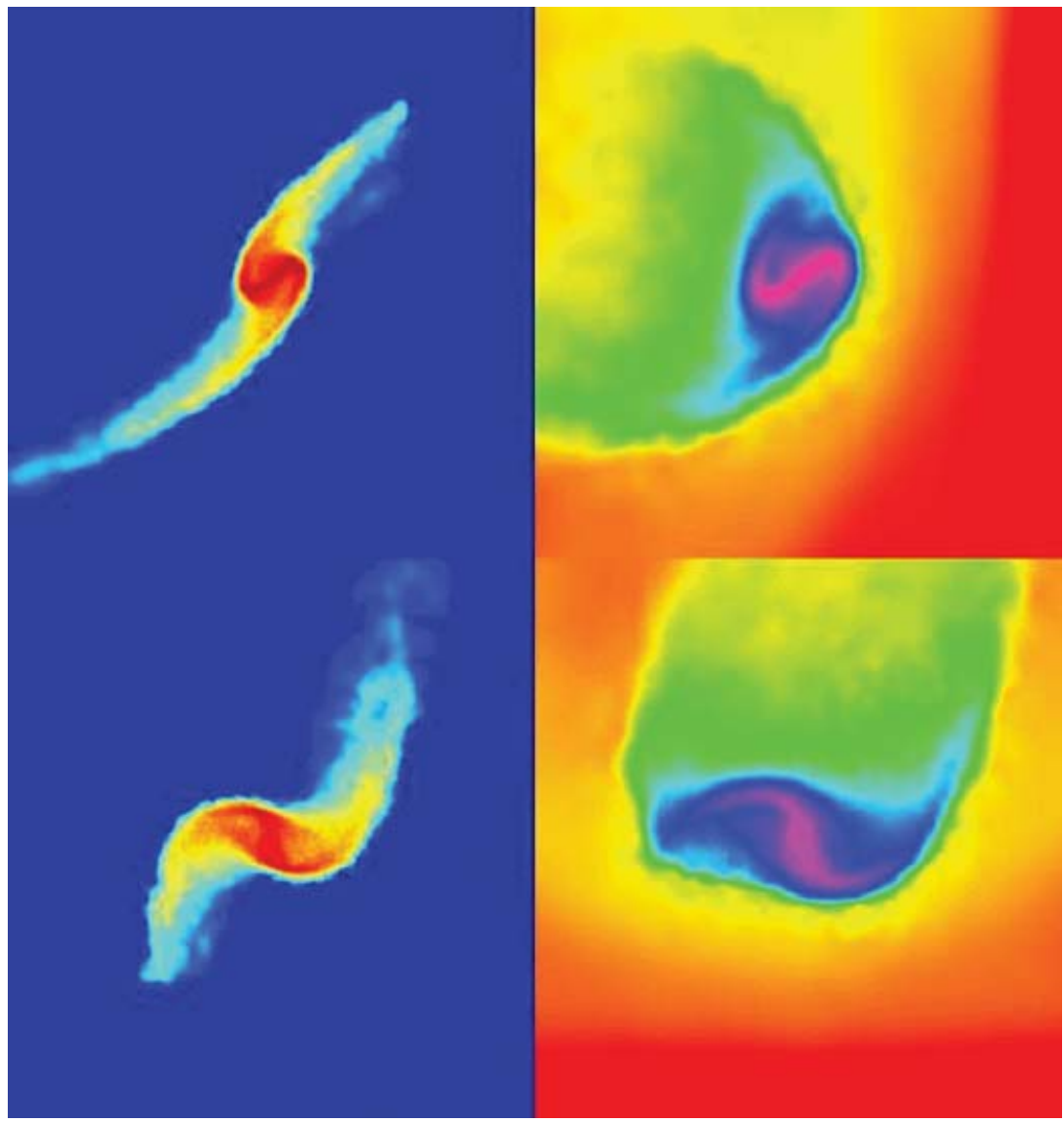

Figure 2. Colour-coded logarithmic density maps for run Rc4aADHR. Projections along (top) and perpendicular to the orbital plane (bottom) are shown for the stars (left) and for the gas component (right). Snapshots are taken at $T=1.7 \mathrm{Gyr}$, while crossing pericenter for the first time. Boxes are $100 \mathrm{kpc}$ on a side for the stars and $30 \mathrm{kpc}$ on a side for the gas (a smaller box is used for the gas to show its structure in greater detail). The two colour maps are such that colours go, respectively, from blue to red through yellow (stars) and from red to magenta through yellow and green (gas) going from lower to higher densities. 


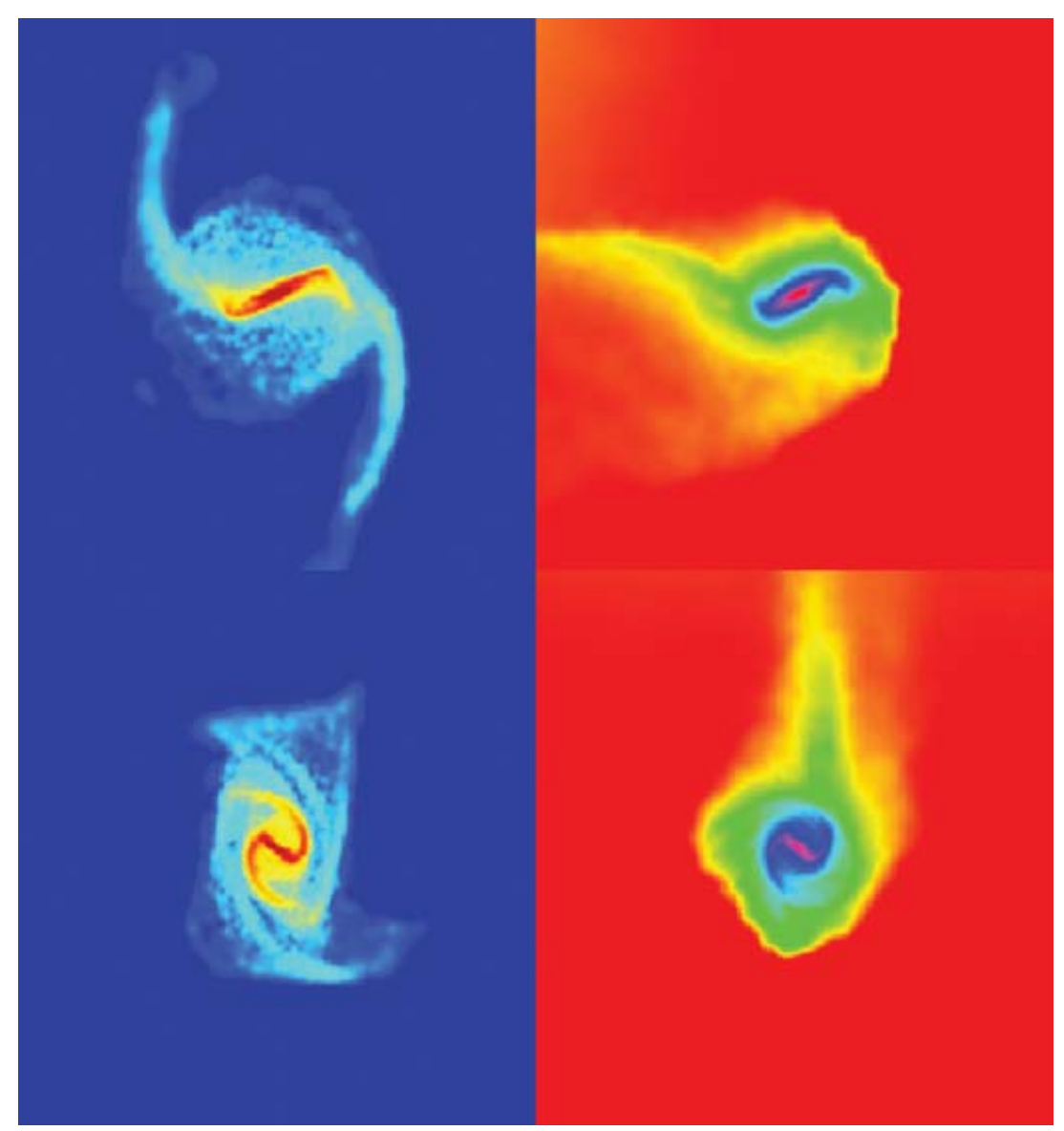

Figure 3. Colour-coded logarithmic density maps for run Rc4aADHR. Projections along (top) and perpendicular to the orbital plane (bottom) are shown for the stars (left) and for the gas component (right). Snapshots are taken at $T=3 \mathrm{Gyr}$, after first pericenter passage. Boxes are $50 \mathrm{kpc}$ on a side.

adiabatic runs, whereas it breaks into clumps pressure confined by the external medium in the cooling runs (see Fig. 7). The evolution of the temperature of the gas is shown in Fig. 9. As the hot halo pushes the gas out of the disc, it also rises its temperature to $\sim 10^{5} \mathrm{~K}$ as a result of compression. If radiative cooling is included, the gas quickly cools down to $10^{4} \mathrm{~K}$; cooling occurs on a time-scale shorter than $10^{7} \mathrm{yr}$ since the gas is heated to temperatures at which the cooling rate is maximum. Occasionally, gas particles that did not gain enough momentum to leave the disc fall back towards the mid-plane as they cool down (compare for example Figs 7 and 8 for run T2c20 - the disc appears more intact at a later time). The distribution of the gas becomes slightly more concentrated towards the mid-plane of the disc (see the evolution of the surface density profiles in Fig. 11), and subsequent stripping is harder. In adiabatic runs, instead, the heated gas remains hot and expands; the gas disc thickens and it is stripped more easily than in the cooling runs (see Fig. 7) as its own pressure support makes it less bound to the disc potential. No stripped gas falls back to the disc.

We consider as 'stripped' all the gas that is removed from the stellar disc. Table 2 also includes the stripping radii found in the simulations, defined as the minimum distance from the centre of the dwarf at which gas particles are stripped. Some of the WT runs were performed twice (see Section 2.2) using a resolution of the background flow equal or higher than that of the FI runs employing the same model. This corresponds to a variation of as much as a factor of 5 in mass resolution (see Section 2.2) and results in a decrease of only $\sim 20$ per cent in the amount of mass lost as the resolution is increased (see Table 2). Moreover, another test shows that a further increase of a factor of 3 in resolution for run T2c20 yields a decrease in mass loss of only 10 per cent (see again Table 2, run T2c20HR). Fig. 12 compares the gas density profiles of the two runs after $0.5 \mathrm{Gyr}$ of evolution. Although complete convergence is not reached, suggesting that some residual two-body heating is present even with more than a million SPH particles, these results show that ram pressure stripping is very weakly dependent on resolution when such large numbers of particles are used. Preliminary results of a convergence study being carried out with several SPH and adaptive mesh refinement (AMR) codes on a ram pressure test without self-gravity indicate that complete convergence is reached with $10^{7} \mathrm{SPH}$ particles (Mayer et al., in preparation). Table 2 reports the gas mass in the disc measured after 0.2 Gyr. The difference in the amount of stripped mass between runs with and without cooling is visibly higher than that between runs adopting different disc inclinations; indeed inclination effects are appreciable only when extreme cases are compared, namely an edge-on versus a face-on run (where the disc inclination is intended relative to the direction of motion of the background gas flow). We note that for the most massive model, V60c4, we have performed only adiabatic runs since our main goal here is to estimate the maximum amount of mass that can be stripped by ram pressure alone. The difference between runs with and without radiative cooling is smaller in terms of stripping radii than it could be guessed 


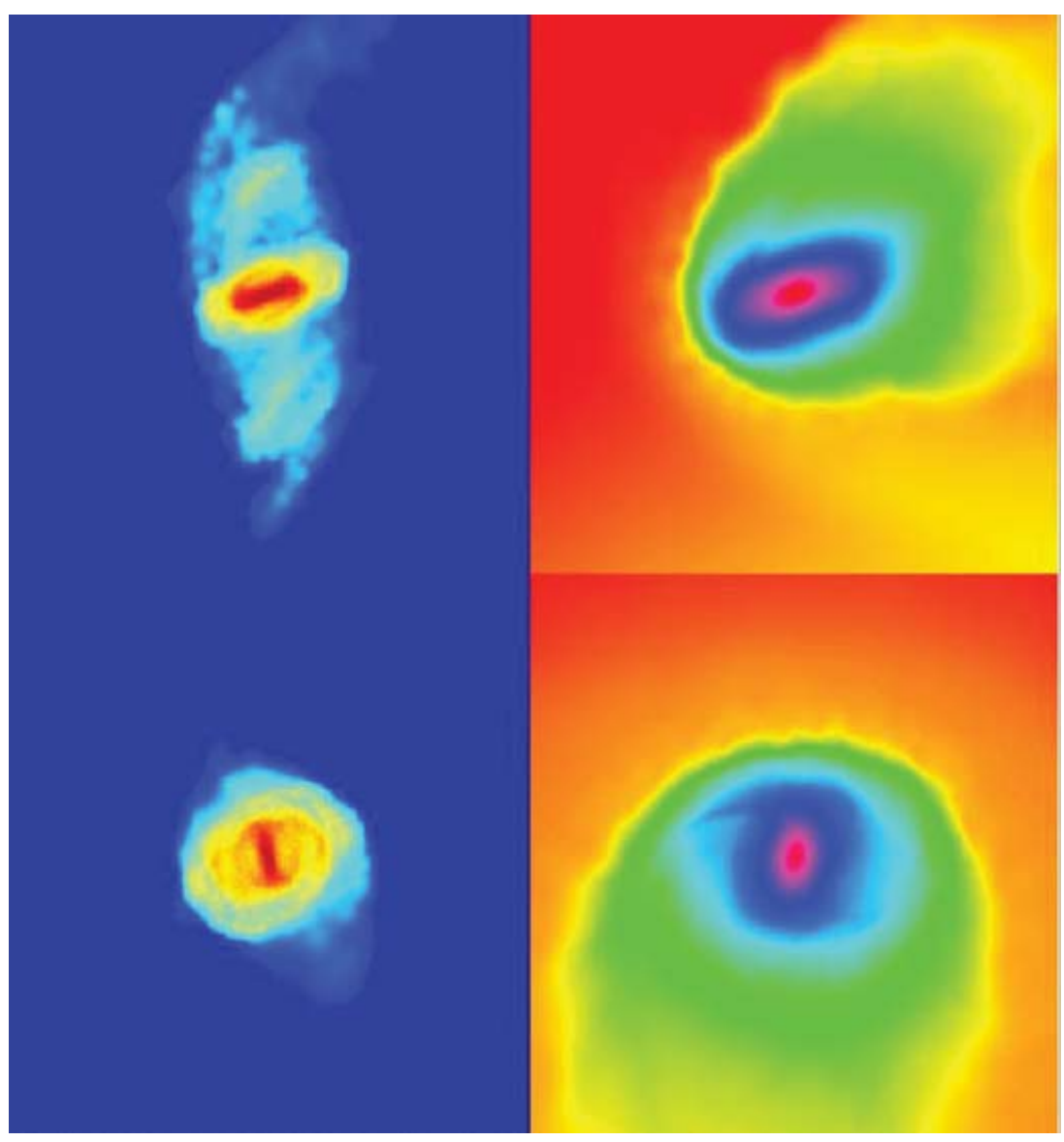

Figure 4. Colour-coded logarithmic density maps for run Rc4aADHR. Projections along (top) and perpendicular to the orbital plane (bottom) are shown for the stars (left) and for the gas component (right). Snapshots are taken at $T=6 \mathrm{Gyr}$, after the second pericenter passage. Boxes are $50 \mathrm{kpc}$ on a side for the stars and $25 \mathrm{kpc}$ on a side for the gas.

from the difference in the amount of stripped mass (Table 2). The reason is that the distribution of gas in the cooling runs becomes more concentrated (thus a given radius contain more mass) as we noted before.

Our results show that model V28c4 is completely stripped in all cases (e.g. Fig. 8) except when cooling and an inclination of $90^{\circ}$ with respect to the flow are used at the same time (both choices go in the direction of minimizing stripping). Instead the models V60c4 and V40c20 always retain a significant amount of gas. This indicates that if the progenitors of dSphs once had massive haloes corresponding to $V_{\text {peak }} \sim 50 \mathrm{~km} \mathrm{~s}^{-1}$, as suggested by recent cosmological simulations (Kravtsov et al. 2004), ram pressure alone was not able to remove their gas early on unless the halo density was once significantly higher than $10^{-4}$ atoms $\mathrm{cm}^{-3}$. On the other hand, these results also suggest that efficient gas removal from ram pressure could have happened at some later stage if tides were able to weaken the potential well of the dwarf bringing $V_{\text {peak }}$ down to $\sim 30 \mathrm{~km} \mathrm{~s}^{-1}$. Interestingly, an NFW halo with $V_{\text {peak }} \sim 30 \mathrm{~km} \mathrm{~s}^{-1}$ or lower does fit the present-day velocity dispersion of dSphs (Lokas 2002; Kazantzidis et al. 2004). We will come back to this in the following sections.

We tried to compare our numerical results with simple analytical estimates of the effectiveness of ram pressure stripping. Gas is removed from the disc of the dwarfs if the ram pressure force is greater than the restoring force per unit area provided by the disc and halo of the dwarf. The condition for ram pressure stripping for an axisymmetric disc system is expressed by (Gunn \& Gott 1972)

$\rho_{\mathrm{h}} v^{2}>2 \pi G \Sigma(R) \Sigma_{\mathrm{g}}(R)$,

where $v$ is the velocity of the galaxy with respect to the surrounding medium, $\rho_{\mathrm{h}}$ is the density of the hot halo of the MW and $\Sigma_{\mathrm{g}}(r)$ is the cold gas surface density at the radius $R$. $\Sigma$ represents the surface mass density of the disc. The minimum radius given by equation (2) is the final stripping radius $R_{\text {str }}$ beyond which the ISM can be removed from the galaxy. Equation (2), however, neglects the contribution from dark matter to the restoring force (see Gavazzi et al. 2001). In all of our models, the dark matter content is high even near the disc; we will simply account for that by replacing $\Sigma(R)$ with $\Sigma_{\mathrm{dm}}+\Sigma_{\mathrm{b}}(R)$, where $\Sigma_{\mathrm{dm}}$ is the surface mass density of the dark matter in a slab with height comparable to the disc thickness and $\Sigma_{\mathrm{b}}(R)$ is the surface mass density of the baryons within the same slab (in our models the gas component always gives a nonnegligible contribution to the disc mass). This formula describes the instantaneous stripping of gas. We found it difficult to use this prescription and reflect both the complex interplay between mechanical and thermodynamical effects seen in the simulations and the fact that the stripping is not exactly instantaneous but continues for some time (typically, it saturates after $\sim 0.2 \mathrm{Gyr}$ ). In particular, $\Sigma_{\mathrm{g}}(r)$ is strongly time dependent due to the action of heating and cooling as we noted above. We find that a reasonable prediction 


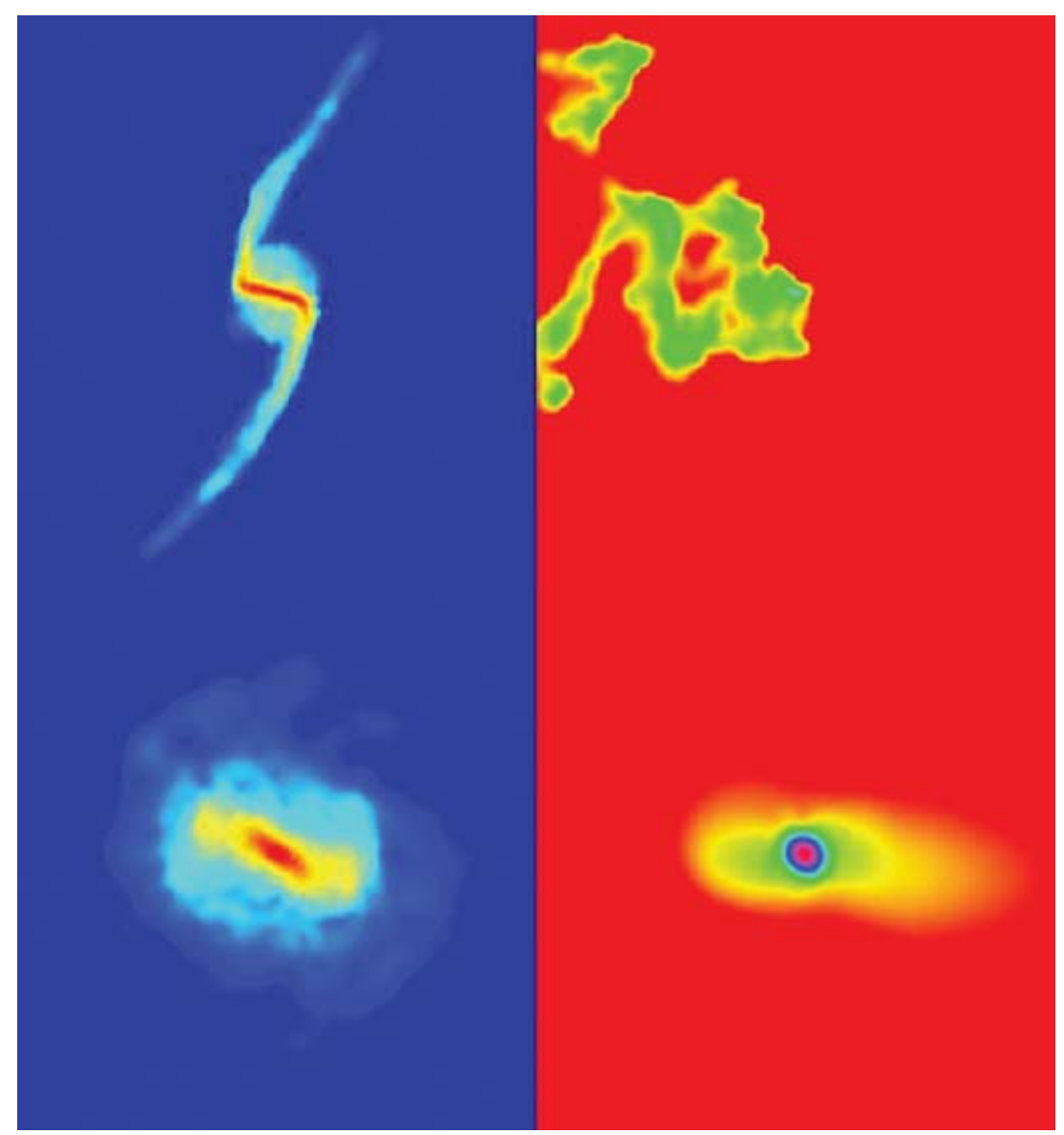

Figure 5. Colour-coded gas density (right) and stellar logarithmic density maps (left) for run Rc4bRC. Projections along the orbital plane are shown in a box of $30 \mathrm{kpc}$ on a side at $T=1.7 \mathrm{Gyr}$ (top) and in a box of $10 \mathrm{kpc}$ on a side at $t=6 \mathrm{Gyr}$ (bottom) after the second pericenter passage. All boxes are centred on the centre of mass of the bound stars. Re-accretion of gas between the two different times has clearly occurred (the gas distribution is completely displaced from the stars at $T=1.7 \mathrm{Gyr}$ ).

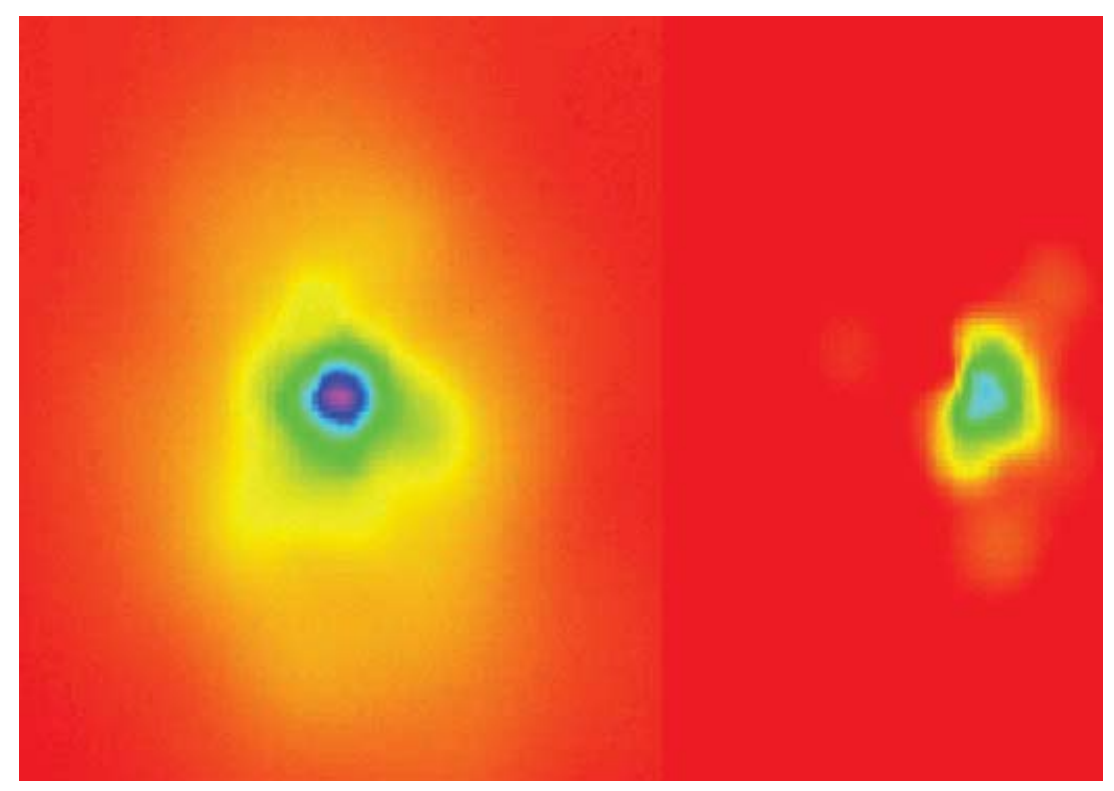

Figure 6. Colour-coded logarithmic gas density maps for run Rc20RC (left) and for run Rc20RCUV (right) at $t=5$ Gyr, after three pericenter passages. The projection along the orbital plane is shown. The boxes are $10 \mathrm{kpc}$ on a side and are centred on the centre of mass of the bound stars. Clearly, the gas remaining in the dwarf is much less and much more diffuse when UV heating is added. 

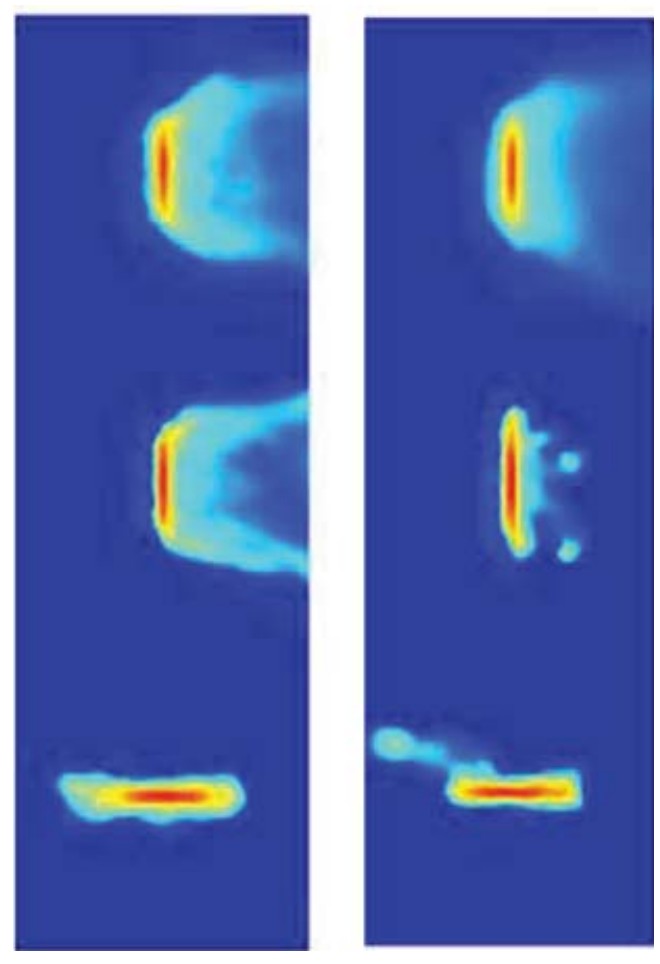

Figure 7. Colour-coded logarithmic gas density map for three WT runs. From top to bottom, an edge-on view after $t=0.05 \mathrm{Gyr}$ (left) and after $t=$ $0.3 \mathrm{Gyr}$ (right) of the gas disc in runs T1c20, T2c20, T3c20. The background gas flows along the horizontal axis from left to right (from right to left in the bottom panel). Boxes are $10 \mathrm{kpc}$ on a side.

can be obtained if we choose not the initial $\Sigma_{\mathrm{g}}(r)$ but that at the time the gas begins to heat up (as little as $10^{6} \mathrm{yr}$ after the beginning of the simulations). The predicted stripping radius (see Table 2) can be 20-30 per cent larger (even for runs with cooling) than that seen at the end of the WT runs, i.e. after $5 \times 10^{8} \mathrm{yr}$. However, the prediction deviates by only 10 per cent from the stripping radius measured in the simulations at earlier times (during the first $10^{8} \mathrm{yr}$ ), i.e. when the approximation of a single, instantaneous stripping event is better satisfied. It is interesting to compare the results of the WT runs with those recently obtained by Roediger \& Hensler (2005) for spiral galaxies inhabiting low-density environments comparable to those considered here. The lowest value of the ram pressure force that they consider, $F_{\mathrm{rp}}=10 \mathrm{~cm}^{-3} \mathrm{~km}^{2} \mathrm{~s}^{-2}$, is comparable to that used in this paper $\left(F_{\mathrm{rp}}=15 \mathrm{~cm}^{-3} \mathrm{~km}^{2} \mathrm{~s}^{-2}\right)$. However, since their galaxy models have a much deeper potential well relative to our galaxies a meaningful comparison requires one to consider their runs with higher ram pressure forces. The gravitational restoring force scales roughly as $V_{\text {peak }}^{2} / r_{\text {peak }}\left(r_{\text {peak }}\right.$ being the radius at which $V_{\text {peak }}$ is reached), where for simplicity we are assuming that the spherical dark halo component provides most of the restoring force [we recall that $V_{\text {peak }}^{2}=G M\left(r<r_{\text {peak }}\right) / r_{\text {peak }}$ ]; it turns out that their galaxies, with $V_{\text {peak }}=160-210 \mathrm{~km} \mathrm{~s}^{-1}$, have restoring forces from 10 to 50 times higher than our galaxies (which have $V_{\text {peak }}=28$ $62 \mathrm{~km} \mathrm{~s}^{-1}$ ). We should then look at those among their runs having $F_{\text {rp }}=100-1000 \mathrm{~cm}^{-3} \mathrm{~km}^{2} \mathrm{~s}^{-2}$ (they do not have runs with $F_{\mathrm{rp}}=$ $500 \mathrm{~cm}^{-3} \mathrm{~km}^{2} \mathrm{~s}^{-2}$ ). These have roughly the same ratio of ram pressure force to gravitational restoring force as our runs. In the latter case, the gas mass loss is comprised between 40 and 95 per cent. In our adiabatic WT runs (cooling is not included in Roediger \& Hensler 2005), we measure a mass loss in the range 40-60 per cent

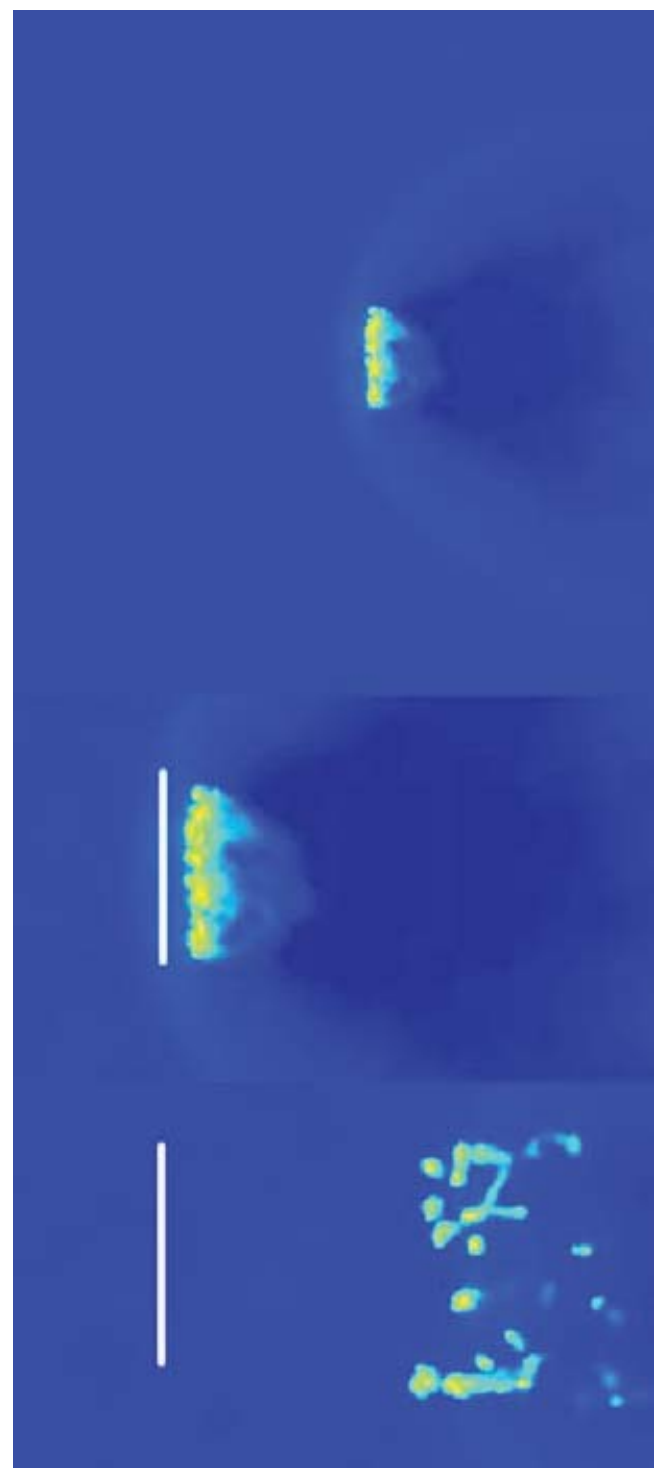

Figure 8. Colour-coded logarithmic gas density map for the gas of the dwarf in run T3c4a. On top, the output after $t=0.05 \mathrm{Gyr}$ is shown in a box of $30 \mathrm{kpc}$ on a side. The bow shock is clearly visible. In the middle panel, we show a zoom-in of the same snapshot (the box is $10 \mathrm{kpc}$ along the longest axis). The bottom panel shows a zoom-in of a snapshot taken after $t=$ $0.3 \mathrm{Gyr}$ (the box is also $10 \mathrm{kpc}$ along the longest axis). The white vertical bar shows the location of the stellar disc. Complete gas stripping occurs in this case. The background gas flows along the horizontal axis from left to right.

for models V60c4 and V40c20, while complete stripping occurs for model V28c4. The fact that our models have slowly rising rotation curves while those of Roediger \& Hensler are very steep in the centre due to the presence of the bulge makes stripping comparatively more efficient in the central regions of our models, which explains why all the gas can be removed in the lightest among our models.

\subsubsection{FI runs}

In these runs, the dwarf galaxy evolves under the combined action of tides and ram pressure. The mass loss as a function of time for a number of runs is shown in Fig. 13. At any given time, we identify 


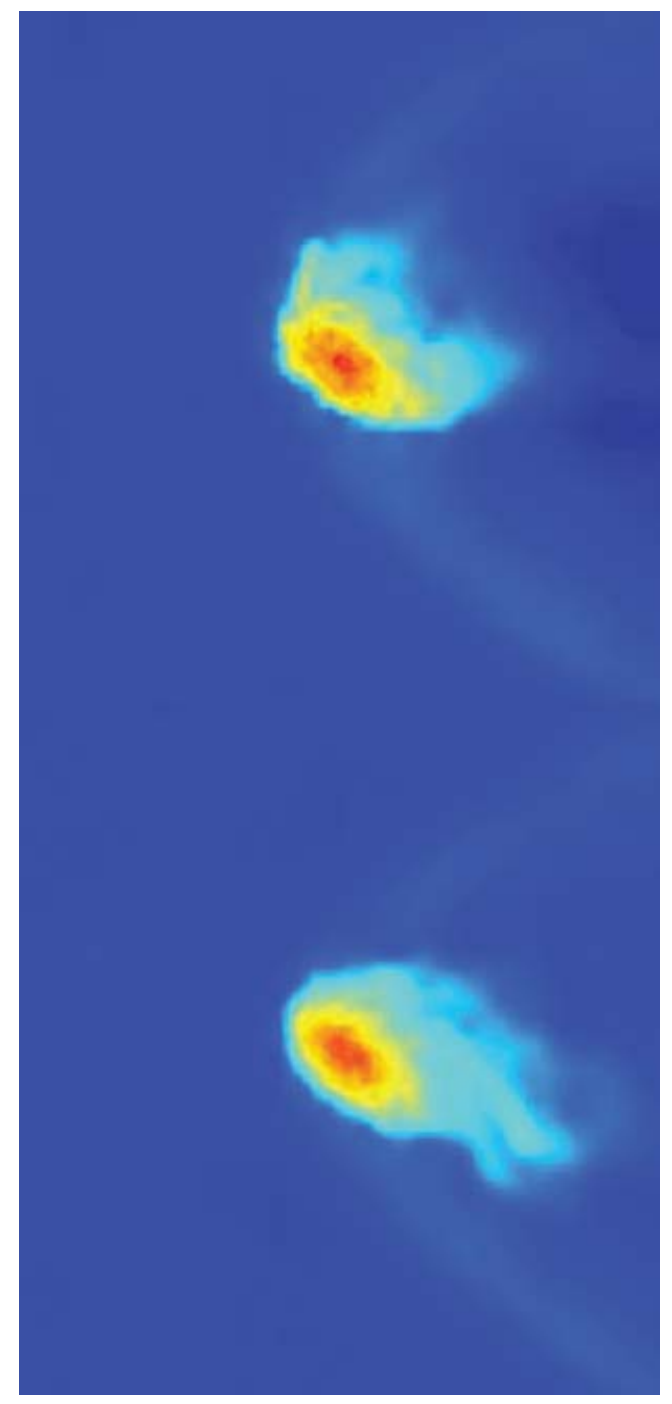

Figure 9. Colour-coded logarithmic gas density map for the gas of the dwarf in run T2c4b. From top to bottom, the outputs after $t=0.05 \mathrm{Gyr}$ and $T=0.3 \mathrm{Gyr}$ are shown. The boxes are $30 \mathrm{kpc}$ on a side. The background flow is along the horizontal axis from left to right.

as bound to the dwarf the gas with temperature $T<T_{\text {vir }}$ (where $T_{\text {vir }}$ is the initial virial temperature of the halo of the dwarf, in the range $\sim 3 \times 10^{4}-10^{5} \mathrm{~K}$ depending on the model) and which is located within the bound stellar component. We checked that by using this definition one essentially selects the gas that is gravitationally bound to the dwarf.

The angle between the direction of the flow in the hot halo and the plane of the disc is continuously changing because of the orbit of the galaxy and since the disc inclination relative to the orbit changes as the dwarf is tidally torqued by the primary. We compare the gas mass losses observed in FI runs with those occurring in the face-on WT runs which yield the highest possible gas mass loss due to ram pressure only. In general, galaxies lose more gas compared to WT runs (compare Table 1 and Table 2, for both adiabatic and cooling runs). However, as we found previously, only in some of the runs (mostly those employing model V28c4) is the dwarf completely stripped of its gas content (see Table 1). Furthermore, while with tides added a dramatic increase in the stripping is always observed in adiabatic runs, stripping can be unexpectedly less effective in cooling runs (compare run Rc4bRC in Table 1 with all the ' $4 \mathrm{a}$ ' runs in Table 2). This happens because the tidally induced bars funnel 20-40 per cent of the initial gas content of the disc within a radius smaller than the initial disc scalelength of the dwarf. The gas shocks through the bar as it loses angular momentum and heats up. In adiabatic runs, this heat cannot be dissipated quickly and the gas ends up more extended. In cooling runs, the gas loses rapidly its thermal energy and there is not enough pressure to stop its inflow. As a result gas profiles become much more concentrated in cooling runs and a larger amount of gas ends up deeper in the potential well where it is harder to strip (Figs 14 and 15). In runs which include the heating from the UV background, the gas remains more extended than with just radiative cooling (Fig. 6) and stripping is much more efficient, with gas mass losses closer to those of adiabatic runs.

Despite the complex interplay between bar instability and thermal physics, it turns out that the fractional amount of stripped mass correlates well with the initial $V_{\text {peak }}$ of the satellite on a given orbit, both with and without cooling (for example, compare run Rc4cRC and run Rc20RC in Table 1, or run Rc4aRCUV and run Rc4bRC). For the same initial $V_{\text {peak }}$, orbits with smaller pericenter lead to a larger reduction in the $V_{\text {peak }}$ due to more numerous and stronger tidal shocks (Fig. 16). This favours ram pressure stripping as it lowers the gravitational restoring force. Furthermore, on orbits with a pericenter of $30 \mathrm{kpc}$, the maximum halo gas density is about a factor of 3 higher relative to the case with a pericenter of $50 \mathrm{kpc}$, and this also favours ram pressure stripping. As a consequence, gas mass loss is significantly larger and faster in runs using a more plunging orbit (Table 1).

Estimating the stripping radius from formula (2) is somewhat meaningless in these runs because the depth and shape of the potential of the dwarf is continuously changing due to the action of tides and the distribution of the bound gas also changes with time. It is noteworthy that the stripping radius estimated using formula (2) with the parameters of the initial models, and using the orbital velocity and background gas density at pericenter, is from two to three times larger than the radius at which most of the bound gas sits in the FI runs after the first pericenter passage, hence after bar formation and inflow. This is because the remaining bound gas is that material which flows to the centre of the bar. On the other hand, if we compare the initial mass of the models within the stripping radius predicted by equation (2) with the mass remaining bound after the first pericenter passage, the agreement improves. The predicted mass is only $30-40$ per cent higher than that measured in the adiabatic simulations. However, in runs with radiative cooling the situation is very different. For the lightest among our models, that should be completely stripped based on Gunn and Gott, some mass can be retained (e.g. run Rc4bRC), and in other cases (e.g. Rc4aRCUV) the remaining bound mass is about 10 times higher than that initially contained within the predicted stripping radius. For adiabatic runs, the reasonable match between the two measurements probably reflects the fact that the largest fraction of the gas is stripped on the first orbit, before the bar affects substantially the evolution (see Fig. 14).

One interesting feature of cooling runs is that sometimes reaccretion of gas is observed. This is clearly shown by the sequence of snapshots in Fig. 5 for run Rc4bRC (which shows that gas that later ends up at the centre of the stellar mass distribution was outside its boundary earlier on) and also in some of the curves describing the evolution of the bound mass with time (top panel in Fig. 13). Fig. 14 also shows, for the same run, that the central gas density 
first becomes lower, just after the first pericenter passage, and then increases considerably. This happens when gas is stripped from the disc near pericenter but does not have enough momentum to completely escape the galaxy. As the galaxy moves towards apocenter, where relative velocities are lower, the gas falls back on to the disc. This is not observed in adiabatic runs because gas that has been removed from the disc quickly becomes hotter than the virial temperature of the dwarf halo, thus being unable to re-accrete.

We repeated several of these simulations without a gaseous halo to isolate the efficiency of gas removal by tides alone. The amount of gas stripped is a factor of 3-6 less than the simulations which include ram pressure stripping, depending on the radiative cooling (Fig. 13). When compared with the WT runs where only ram pressure is included, tides strip from similar to appreciably lower amounts of mass (lower panel of Fig. 13). The relative increase of stripping when ram pressure is added to tides is maximum for model V40c20, that has the steepest rise of the dark matter density in the centre (see Fig. 1). This reflects the different physical scalings of ram pressure and tidal forces. In these highly concentrated haloes, the response to tidal shocks is nearly adiabatic near the centre (Taffoni et al. 2003; Kazantzidis et al. 2004), hence stripping is expected to be minimal inside $R_{v=v_{\text {peak }}}$. However, this is applicable only to stripping induced by tidal shocks; the time-scale on which instantaneous ram pressure acts is instead much smaller than that of tidal shocks, $<10^{7} \mathrm{yr}$ (we follow Mori \& Burkert 2000 for parameters of e.g. run Rc20V60RC) against $\sim 10^{8}$ yr $\left(\sim R_{\text {peri }} / V_{\text {peri }}\right)$. Such time-scale is also smaller than the local gas orbital time even in a highly concentrated halo $\left(>10^{7} \mathrm{yr}\right.$ ) hence the response of the galaxy to ram pressure is impulsive instead of adiabatic. On the other hand, the other models, having low-concentration haloes, respond more impulsively to tides in the first place, so that the increase in stripping efficiency is not so dramatic.

In these simulations, the peak velocity of the rotation curve of the satellites, $V_{\text {peak }}$, drops by 20-50 per cent after several orbits (Fig. 16), while in cosmological simulations it often decreases by more than a factor of 2 over several Gyr (Kravtsov et al. 2004, but see Ghigna et al. 1998). Such difference may be due to the limited number of orbital configurations here explored (some of the satellites in Kravtsov et al. 2004 have pericenters even smaller than $30 \mathrm{kpc}$ ) and/or to the fact that cosmological runs do not include baryons and do not have enough force and mass resolution to follow the complex disc evolution, especially bar formation and the resulting gas inflows that can produce a remarkable increase in the central density (our force resolution is five times better than the best published cosmological simulations with baryons). These issues will be investigated systematically in a forthcoming paper (Kazantzidis et al., in preparation).

In summary, the final gas fractions drops to 0-50 per cent of the initial gas content for galaxies on orbits with pericenter of $50 \mathrm{kpc}$, with 10 per cent being a typical fraction, and to as low as $0-6$ per cent of the initial values for galaxies on an orbit with pericenter of $30 \mathrm{kpc}$ (see Table 1). In the runs that include the effect of the cosmic UV background even when there is gas left inside the dwarf this is ionized, and the observational upper limit on the mass of neutral hydrogen in dSphs, $M_{\mathrm{HI}}<0.01 M_{\mathrm{s}}$, where $M_{\mathrm{s}}$ is the stellar mass, is fully satisfied. The gas will be able to recombine later on as the cosmic background fades away, but the local UV radiation flux from the Milky Way or M31 might be enough to keep it ionized even at low redshift (Mashchenko et al. 2004). The current upper limits on the total amount of gas in dSphs including the ionized component are much higher, $M_{\mathrm{g}}<0.1 M_{\mathrm{s}}$ (Gallagher et al. 2003), and are satisfied in the final states of the dwarfs for the majority of our runs. Hence, a combination of ram pressure and tidal stripping succeeds in explaining how dwarf spheroidals have little or no neutral hydrogen today. On any given orbit, dwarf models with higher $V_{\text {peak }}$ are stripped less severely and, in particular, our most massive model, V60c4 $\left(V_{\text {peak }}=62 \mathrm{~km} \mathrm{~s}^{-1}\right)$, always retains a substantial amount of gas. This is consistent with the fact that bright dwarf elliptical (dE) satellites of M31, like NGC 205, whose central velocity dispersion, $\sigma=30-45 \mathrm{~km} \mathrm{~s}^{-1}$, is comparable to that of the evolved states of model V60c4, do have some amount of neutral gas (Mateo 1998).

When neutral gas remains in the dwarfs, most of it will be rapidly turned into stars as it reaches very high densities due to the effect of the bar, and bursts localized near the centre of the dwarf are expected at pericenter passages, as described in Mayer et al. (2001a). Hence, we predict that the higher the mass of the dwarf (not its luminosity) the more extended its star formation history should be. For dwarfs that start out with similar masses, our simulations suggest that the outcome will vary depending on their orbit.

A signature in the star formation history of the dwarfs is expected at the epoch when it underwent the first close approach within the Milky Way. This can be either a burst or a marked depression of the star formation depending on whether just a fraction or most of the gas is removed from the dwarf galaxy.

\subsection{Kelvin-Helmholtz instabilities and turbulent stripping}

Gas that survives instantaneous ram pressure can be stripped by the Kelvin-Helmholtz (KH) instability developing at the interface between the gas of the dwarf galaxy and the ISM (Nulsen 1982; Murray et al. 1993; Quilis, Moore \& Bower 2000). This is also known as turbulent stripping since the gas reaches a turbulent state as the instability develops. In order to develop, $\mathrm{KH}$ instabilities require a sharp boundary to exist between the two fluids. Even at the high resolution adopted here, SPH is hardly capable of resolving $\mathrm{KH}$ instabilities mostly because the artificial viscosity and the fact that densities are smoothed in SPH both contribute to 'blur' any sharp interface.

An approximate 'effective' local kinematical viscosity can be calculated as $v=\alpha c_{\mathrm{s}} h / 8$, where $\alpha$ is the coefficient of the linear term in the artificial viscosity, $c_{\mathrm{s}}$ is the sound speed and $h$ is the SPH smoothing length (Lufkin et al. 2004). The Reynolds number is then $R e \sim L v_{\text {gal }} / v$, where $L$ is thetypical size of the object moving in an external medium (the disc of the dwarf here) and $v_{\text {gal }}$ is the speed at which it is moving; this number is strictly a function of position (since $v$ is a function of position) and is in the range 20-100 in our runs (the highest Reynolds numbers occur in the highest resolution WT runs). This is of course much lower than the Reynolds numbers typical of turbulent flows (up to $10^{4}$ ). Aside from numerical issues that might hamper the development of $\mathrm{KH}$ instabilities in the simulations, we can ask whether turbulent stripping is likely to be important for dwarf galaxies moving in a very diffuse halo as those that we are modelling here. Mori \& Burkert (2000) found that spherical dwarf galaxies moving at a few thousand $\mathrm{km} \mathrm{s}^{-1}$ in a dense cluster hot halo will be stripped on a time-scale significantly smaller than the crossing time of the galaxy in the cluster. Following Mori \& Burkert (2000), we use the following equation to obtain an estimate of the expected characteristic time-scale for stripping by $\mathrm{KH}$ instabilities including the stabilizing effect of gravity 

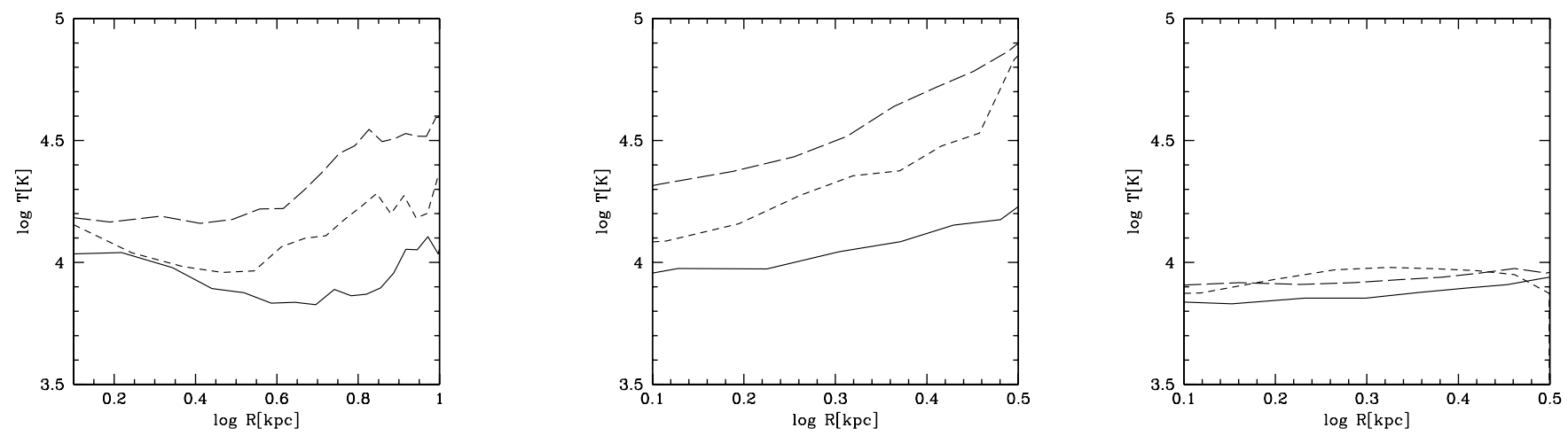

Figure 10. Evolution of temperature profiles of the gas in WT runs. Profiles are azimuthally averaged in a cylinder with height twice that of the stellar disc $(R$ is the cylindrical radius). The solid line is for $t=0.02 \mathrm{Gyr}$, the short dashed line is for $t=0.1 \mathrm{Gyr}$ and the long dashed line is for $t=0.3 \mathrm{Gyr}$. From left to right, run T1c4b, T1c20 and T2c20 are shown.
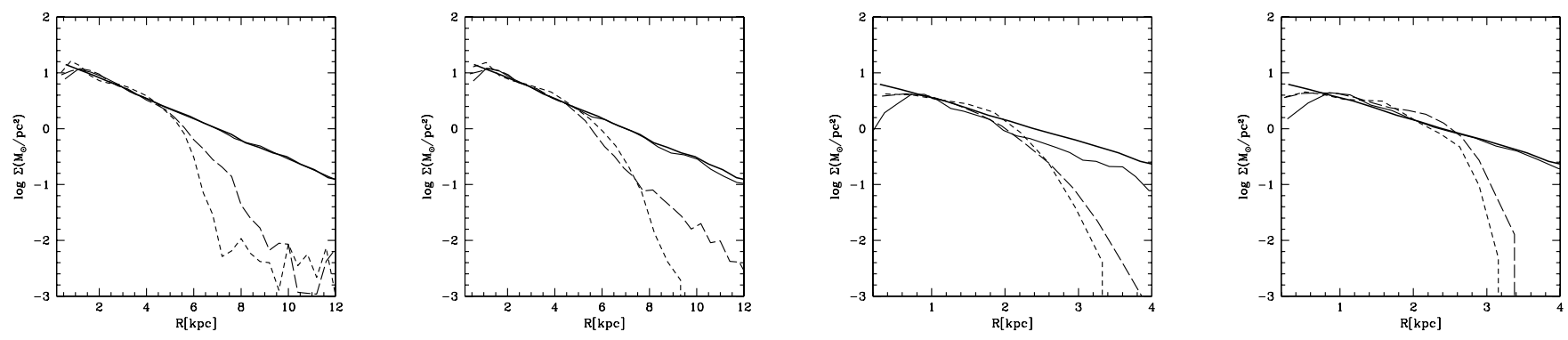

Figure 11. Evolution of density profiles of the gas in WT runs. Profiles are azimuthally averaged in a cylinder with height equal to that of the stellar disc $(R$ is the cylindrical radius). The thick solid line is for $t=0$, the thin solid line is for $t=0.02 \mathrm{Gyr}$, the short dashed line is for $t=0.1 \mathrm{Gyr}$ and the long dashed line is for $t=0.3$ Gyr. From left to right, runs T1c $4 \mathrm{~b}, \mathrm{~T} 3 \mathrm{c} 4 \mathrm{~b}, \mathrm{~T} 1 \mathrm{c} 20$ and T2c20 are shown.

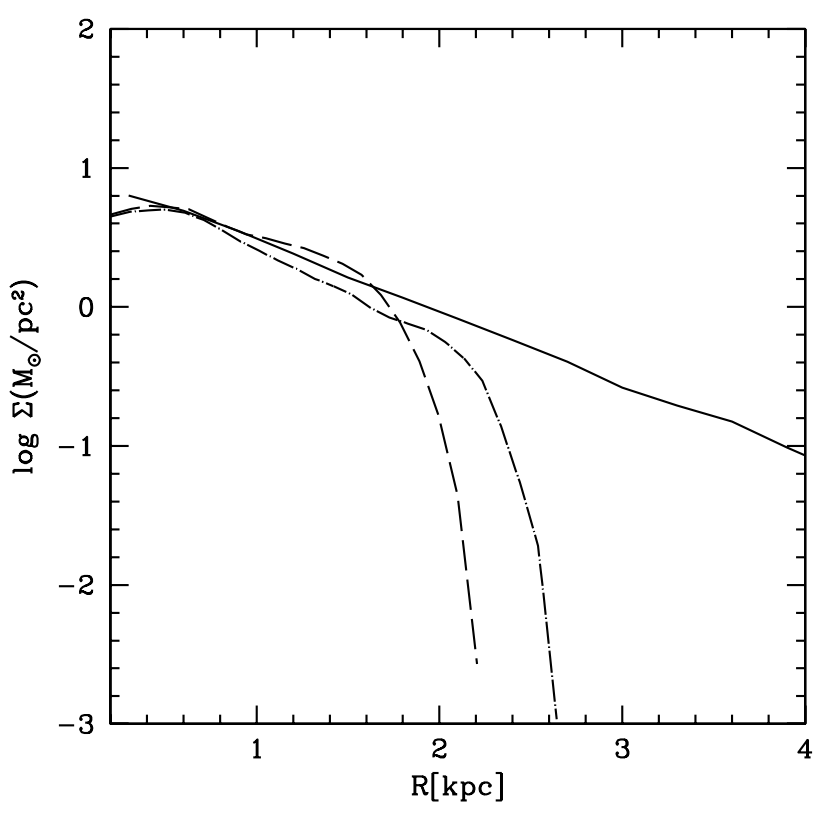

Figure 12. Evolution of density profiles of the gas in WT runs with cooling employing two different resolutions (runs T2c20 and T2c20HR). Profiles are azimuthally averaged in a cylinder with height equal to that of the stellar disc ( $R$ is the cylindrical radius). The thick solid line is for the gas profile at $t=0$ (this being the same for both runs), the long dashed line is for run T2c20 at $t=0.5 \mathrm{Gyr}$ and the dot-dashed line is for run T2c20HR at the same time. (which is mainly provided by the dark halo potential in our models):

$$
\begin{aligned}
& \tau_{\mathrm{KH}}=\frac{F M_{0}}{\dot{M}_{\mathrm{KH}}}, \\
&=2.19 \times 10^{9}\left(\frac{F}{0.1}\right)\left(\frac{M_{0}}{10^{9} \mathrm{M}_{\odot}}\right)^{\frac{1}{7}}\left(\frac{n_{\mathrm{h}}}{10^{-4} \mathrm{~cm}^{-3}}\right)^{-1} \\
& \times\left(\frac{v_{\mathrm{gal}}}{10^{3} \mathrm{~km} \mathrm{~s}^{-1}}\right)^{-1} \mathrm{yr} .
\end{aligned}
$$

The equation assumes spherical symmetry, which is a reasonable assumption since our galaxies are dominated by a spherical dark matter halo. In the equation, $M_{0}$ is the CDM halo mass within the radius $R_{\text {strip }}$ down to which galaxies are stripped in the WT runs (see Table 2; these simulations offer a cleaner test case since they include only ram pressure), $F=M_{\text {bar }} / M_{0}$ is the mass ratio between the baryons and the CDM halo within the same radius and $\dot{M}_{\mathrm{KH}}=\pi R_{\text {strip }}^{2} \rho_{\mathrm{h}} v_{\text {gal }}$ is the mass-loss rate from the galaxy through $\mathrm{KH}$ instabilities $\left(\rho_{\mathrm{h}}\right.$ is the hot halo density and $v_{\text {gal }}$ is the velocity of the galaxy through the hot halo). For the gas densities $\left(\sim 10^{-4}\right.$ atoms $\mathrm{cm}^{-3}$ ) and velocities occurring at pericenter, the time-scale for $\mathrm{KH}$ stripping is, respectively, $4 \mathrm{Gyr}$ for model V40c20 and $10 \mathrm{Gyr}$ for model V60c4, these being the model galaxies in which instantaneous ram pressure is not capable of removing all the gas. For the gas densities and velocities where the satellites spend most of their time, namely at distances larger than $100 \mathrm{kpc}$, the $\mathrm{KH}$ stripping time-scale is at least several Hubble times. We verified that our conclusion on the negligible effect of $\mathrm{KH}$ stripping holds even 

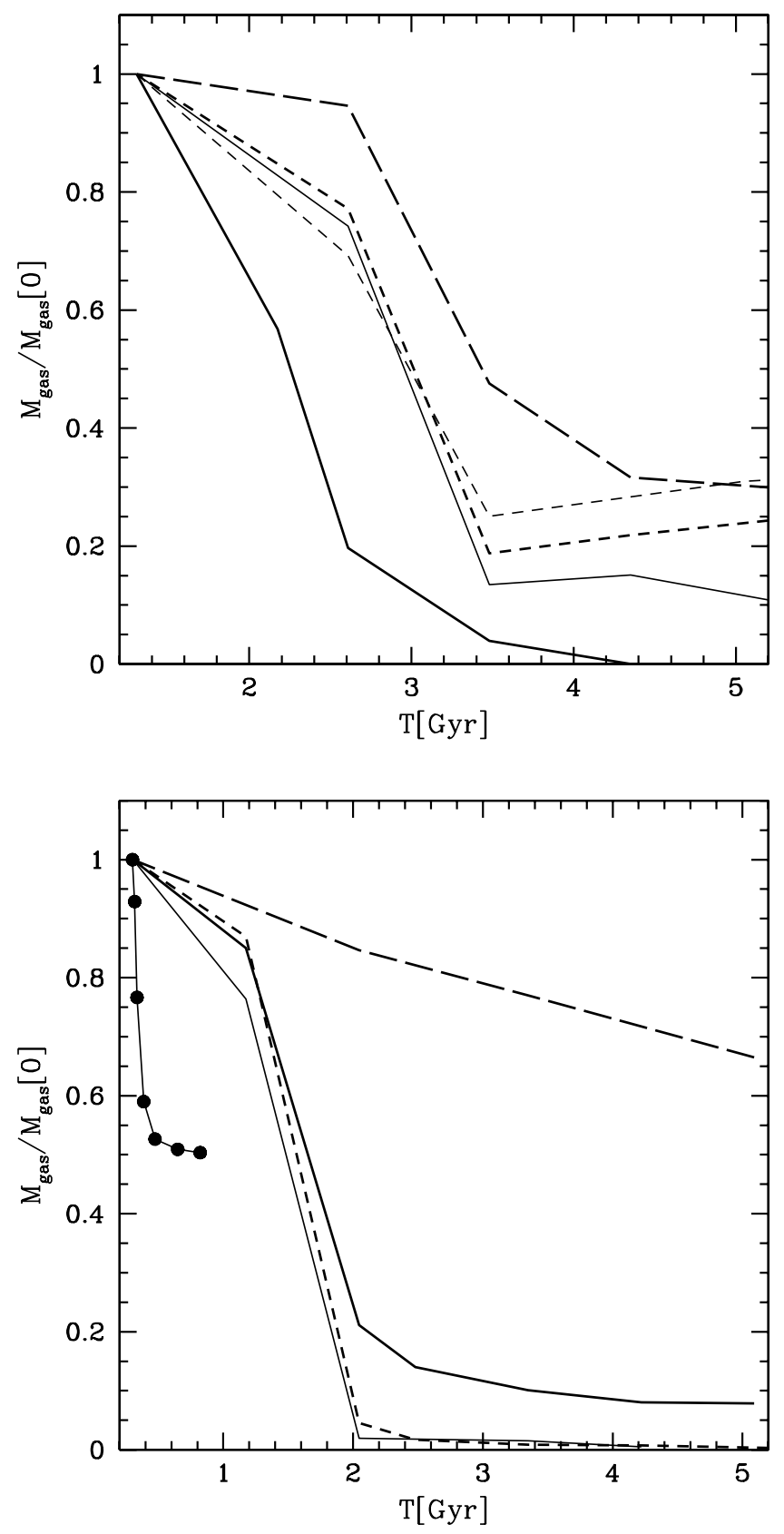

Figure 13. Time evolution of the bound gas mass for FI runs using models V60c4, V28c4 (top) and runs using model V40c20 (bottom). Top: run Rc4aAD (thin solid line), Rc4aRCUV (thin short dashed line), Rc4bTD (long dashed line), Rc4bAD (thick solid line), Rc4bRC (thick short dashed line). Bottom: run Rc20TD (long dashed line), Rc20AD (thin solid line), Rc20RC (thick solid line), Rc20RCUV (short dashed line). The line with dots shows the mass-loss rate (due to ram pressure only) for one of the WT runs, T1c20 (see Table 2 - the curve stops at less than 1 Gyr since WT runs were run for a short time-scale since stripping saturates very early on).

when we use other estimates, such as that in Murray et al. (1993), who look at the critical gravitational acceleration threshold needed for stability against low-order (long wavelength) modes. Moreover, radiative cooling can weaken considerably $\mathrm{KH}$ instabilities even when self-gravity is absent or low; in this case, the instability is suppressed only locally and a core-halo structure is produced such that the core is not stripped (Vietri, Ferrara \& Miniati 1997).
Although even this second type of stabilization should be present in our runs, we expect it to be of secondary importance since the net radiative losses in our simulations are significantly lower than those assumed by Vietri et al. (1997), especially in the runs including the UV background radiation.

Shear viscosity in a laminar flow $(R e<30)$ can also produce a slow stripping of the gas with time-scales and mass-loss rates comparable to those of $\mathrm{KH}$ stripping in the turbulent regime (Nulsen 1982). Physical viscosity is not included in our treatment of SPH and therefore this viscous stripping mode is missing. Spurious viscous stripping from the artificial viscosity term is limited by our reasonably high resolution and the Balsara correction. The characteristic viscous time is $\tau=L^{2} / v$ is $\sim 10 \mathrm{Gyr}$, which means viscosity effects are negligible given that the important time-scales, those related to ram pressure and tidal shocks, are much smaller (between $10^{7}$ and $10^{8} \mathrm{yr}$ ). Tittley, Pearce \& Couchman (2001) have shown that SPH can alter the magnitude and scaling with density of hydrodynamical drags because of the way pressure forces among neighbouring cold and hot background particles are calculated (in essence the cross-section of an object moving in a hot medium can be artificially enhanced or decreased). However, the effect is particularly severe, enhancing the drag over the expected value, only in the subsonic regime, whereas our galaxies all move at slightly supersonic velocities.

In summary, our simulations have enough resolution to overcome artificial viscous stripping but cannot model correctly turbulent stripping yet. However, although SPH is often blamed for its inability to model hydrodynamical turbulence, we recall that published grid simulations are of similar quality when it comes to the ability to model turbulence - Marcolini et al. (2003) find Reynolds numbers similar to what we find.

\subsection{Dark and baryonic contents of the remnants: can we explain the faintest $\mathrm{dSphs}$ ?}

In FI runs, the stellar disc is stripped and transformed into a spheroidal system by means of tidal heating and bar-buckling instabilities as described in Mayer et al. (2001a,b). The ratio $M_{\text {dark }} / M_{\text {bar }}$ between dark matter mass and baryonic mass in the final objects varies between 3 and 12, the highest values occurring in runs with model V40c20. In remnants with the highest dark matter contents or highest central concentration of gas, the buckling instability is weaker, with the result that the bar-like shape is preserved. The trend with gas concentration is consistent with the findings of Debattista et al. (in preparation) for isolated bar-unstable galaxies with gas. However, in reality the central gas would form stars and its distribution will become less concentrated. The final central $v / \sigma$ of the stars is below 0.5 , thus compatible with those of dSphs, and the central final velocity dispersions are in the range $10-30 \mathrm{~km} \mathrm{~s}^{-1}$.

We can ask whether the remnants of model, V40c20, can reproduce the overall properties of the most extreme dSphs in terms of low luminosity and high $\mathrm{M} / \mathrm{L}$. Example of such objects are Draco, Ursa Minor and And IX (Kleyna et al. 2005). In adiabatic runs, the gas is completely stripped after the first pericenter passage. The observed truncation of the star formation history in Draco and Ursa Minor could be interpreted as the result of their infall into the Milky Way halo. However, in runs with cooling some gas is retained. Assuming a star formation rate comparable to that deduced for Draco and Ursa Minor by Hernandez et al. (2000), 100-200 $\mathrm{M}_{\odot} \mathrm{Myr}^{-1}$, we obtain that the remaining gas in run Rc20RC will be turned into stars in about 5 Gyr, leaving a dwarf completely devoid of gas. However, this implies a fairly extended star formation history, contrary to what 

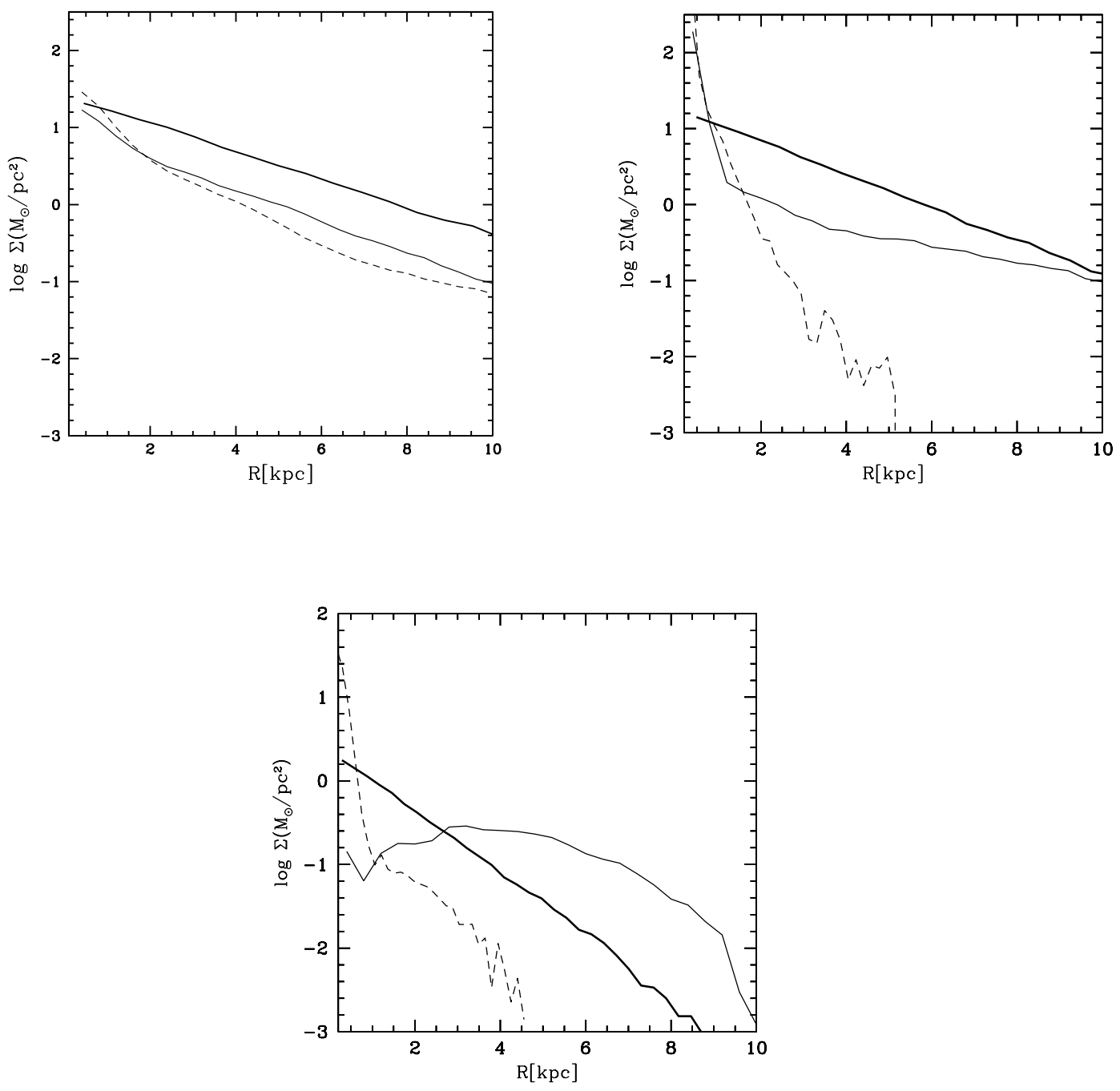

Figure 14. Evolution of gas surface density profiles in FI runs. The thick solid line is used for the initial profile, the thin solid line is used for the profile at $T=3 \mathrm{Gyr}$ (after first pericenter passage) and the dashed line is used for the profile at $T=6 \mathrm{Gyr}$ (after second pericenter passage). From top left to bottom, the runs Rc4aAD, Rc4aRCUV and Rc4bRC are shown. The unusual shape of the profile at intermediate times in the bottom panel reflects the fact that gas is displaced from the galaxy at this point (see also Fig. 5).
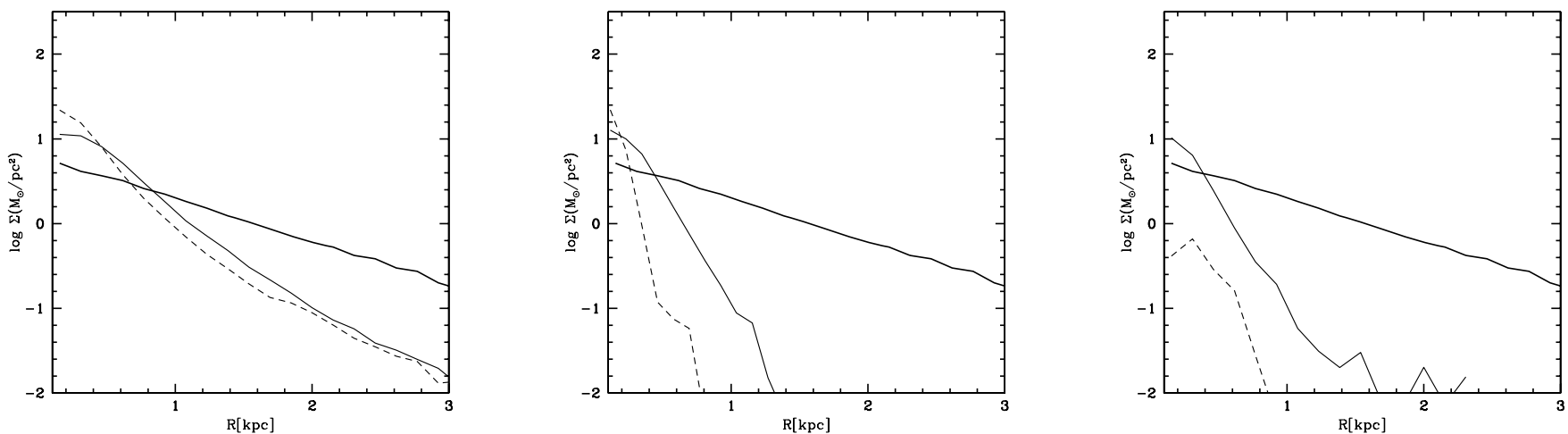

Figure 15. Evolution of gas surface density profiles in FI runs. The thick solid line is used for the initial profile, the thin solid line for the profile after first pericenter crossing $(T=1 \mathrm{Gyr})$ and the dashed line for the profile after second pericenter passage ( $T=3 \mathrm{Gyr})$. From left to right, run Rc20TD, Rc20RC and RC20RCUV are shown.

observed for Draco, and the amount of gas that needs to be turned into stars is non-negligible, $\sim 6 \times 10^{6} \mathrm{M}_{\odot}$; if we sum such mass to the remaining bound stellar mass, we get $5 \times 10^{7} \mathrm{~L} \odot$. For a M/L $\sim 4$, as typical of old stellar populations we would get a luminos- ity $\sim 10^{7} \mathrm{M}_{\odot}$, i.e. 50 times higher than the $V$-band luminosity of Draco (Odenkirchen et al. 2001). Since the largest fraction of the final baryonic mass is contributed by the initial stellar mass in the model (tidal stripping is moderate in highly concentrated haloes), 


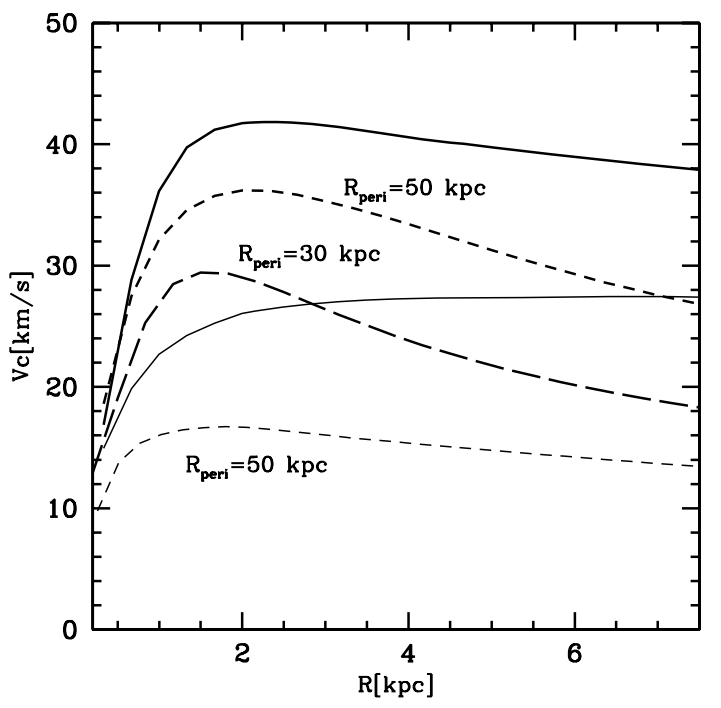

Figure 16. Evolution of the rotation curves of the dwarfs (including the contributions of both dark matter and baryons) in run Rc20TD (thick lines)and run Rc4aTD (thin lines). The solid lines represent the initial conditions, while the short dashed show the curves after three orbits, corresponding to $t=4 \mathrm{Gyr}$ and $t=10.2 \mathrm{Gyr}$, respectively. The thick long dashed line shows the curve for run Rc20bAD after $t=10.2$ Gyr. Pericenter distances for the various orbits are indicated in the plot. Note that runs Rc20TD and runs Rc20bAD employ the same initial dwarf model but different orbits.

an excessive final luminosity would result also in adiabatic runs. As for the dark matter content, one would obtain $\mathrm{M} / \mathrm{L} \sim 50$, as high as typical estimates for $\mathrm{M} / \mathrm{L}$ in Ursa Minor and comparable to the lower limits for M/L in Draco (Mateo 1998; Wilkinson et al. 2004) but too low to match the M/L estimated for And IX ( $>100$; see Kleyna et al. 2005). The final projected central velocity dispersion, $\sim 15 \mathrm{~km} \mathrm{~s}^{-1}$, is consistent with the peak velocity dispersion of Draco (Wilkinson et al. 2004). The central dark matter density measured for the stirred dwarf at the end of the simulation, $0.065 \mathrm{M}_{\odot} \mathrm{pc}^{-3}$ is also in rough agreement with the value estimated for Draco $\left(\sim 0.1 \mathrm{M}_{\odot} \mathrm{pc}^{-3}\right)$. Thus, run Rc20RC produces a model with roughly the right dark matter content but an excessive baryonic content. Adding heating and photoionization from a uniform UV background (run Rc20RCUV) aids gas removal and leaves essentially no neutral gas after a few Gyr. In this case, the star formation history, as in the adiabatic runs, would have been essentially truncated after the first close encounter with the Milky Way. Nevertheless, the total stellar mass of the dwarf would still yield luminosity about an order of magnitude higher than that of Draco.

The final luminosity depends on the initial stellar and gas content of the model, which was chosen to be fairly representative of the baryonic content of today's dIrrs. Although Draco likely had a halo massive enough to resist gas removal from supernovae feedback or photoionization, the progenitors from which it assembled at very high redshift were certainly much smaller and were likely affected by these mechanisms. Hence, one can imagine that the main progenitor of Draco had a baryon fraction much lower than the one we used here. In such a scenario, however, environmental effects would not have played a primary role in setting the current high $\mathrm{M} / \mathrm{L}$ of Draco. One alternative explanation is to imagine that Draco was a mostly gaseous disky dwarf by the time it entered the Milky Way, with minimal star formation having taken place. Such a low star formation rate can be simply the result of an extended, relatively high angular momentum disc being almost everywhere Toomre sta- ble and below the density threshold necessary for star formation to happen, as observed today in some dIrrs and LSB galaxies (Verde, Oh \& Jimenez 2002). To explore this possibility, we have run a simulation in which the initial model has the same structural properties of model V40c20 except that now as much as 90 per cent of the disc is gaseous. A weaker stellar bar forms given the lower stellar mass which produces a weaker gas inflow, and a larger relative fraction of the disc mass is stripped by ram pressure. In an adiabatic run, all the gas is stripped after two pericenter passages, leaving a gas-free remnant that roughly matches the luminosity of Draco after $\sim 3 \mathrm{Gyr}$. In a run with cooling and the cosmic UV radiation of the order of $10^{5} \mathrm{M}_{\odot}$ of gas remain in the dwarf; however, all this gas is ionized and no star formation would be possible, and even in this case the final stellar mass would be comparable to that of Draco.

\subsection{Fate of stripped gas}

The stripped gas has a very different fate in the adiabatic and in the cooling runs. In the adiabatic runs, it ends up quite diffuse and hot $\left(T>10^{5} \mathrm{~K}\right)$ and quickly thermalizes and mixes with the gas in the MW halo. When cooling is allowed, the gas along the tails fragments into dense clouds and sheet-like structures pressure confined by the ambient medium. This is shown in Fig. 17. These clouds remain cold $\left(T<10^{5} \mathrm{~K}\right)$ until they are dissolved as they sink deeper in the potential well due to gas drag. Sinking of the gaseous tails is marginal in the adiabatic runs because the stripped gas is too diffuse to experience significant drag. Fragmentation is suppressed in the first place in adiabatic runs because in the absence of cooling density fluctuations along tails are rapidly dissipated. In the simulations with UV heating, clouds are also present, although they are slightly more diffuse and hotter (see Fig. 17).

Individual clouds survive for a time-scale of $\sim 10^{8} \mathrm{yr}$. The dissolution of the clouds is due to a combination of ram pressure and tidal disruption, but spurious evaporation due to artificial viscosity and discreteness effects (particle-particle collisions) may be important, especially for the smallest clouds that are close to the resolution limit (we consider a cloud to be unresolved if it contains less than 64 particles, i.e. twice the number of neighbouring particles used in the SPH calculation). We calculate that ablation of the clouds due to $\mathrm{KH}$ stripping would occur on time-scales comparable to the dissolution time-scales observed in the simulations.

A single stripped satellite produces tens of blobs with masses in the range $10^{4}-10^{6} \mathrm{M}_{\odot}$ and sizes in the range $0.5-5 \mathrm{kpc}$. These blobs could explain the cold Hi fragments identified by Thilker et al. (2004) around M31. These clouds have column densities $\left(\sim 10^{20} \mathrm{~cm}^{-2}\right)$ and masses (if placed at roughly the distance of M31) of $10^{5}-10^{6} \mathrm{M}_{\odot}$, similar to the most massive among our clouds. They have different velocities with respect to the systemic velocities, which lead Thilker et al. to suggest that they could be hardly tidal debris of disrupted dwarfs. However, a spread in velocities arises naturally because of the drag exerted by the diffuse gaseous halo. We measure velocities differing by up to a factor of 2 among the clouds in our simulations. The variety of shapes of the clouds detected by Thilker et al., from nearly spherical to elongated filaments, is also reproduced by our simulations (Fig. 17). The clouds do not appear tidally disturbed because they are pressure confined. However, their origin is partly tidal since they are the result of the combined removal of gas by tides and ram pressure. Although our simulations lack lowtemperature cooling (by molecules and metals), the heating from the cosmic or local UV radiation should keep their temperature and densities close to the values observed in the simulations. However, since many of the clouds are just above our resolution limit, we feel 

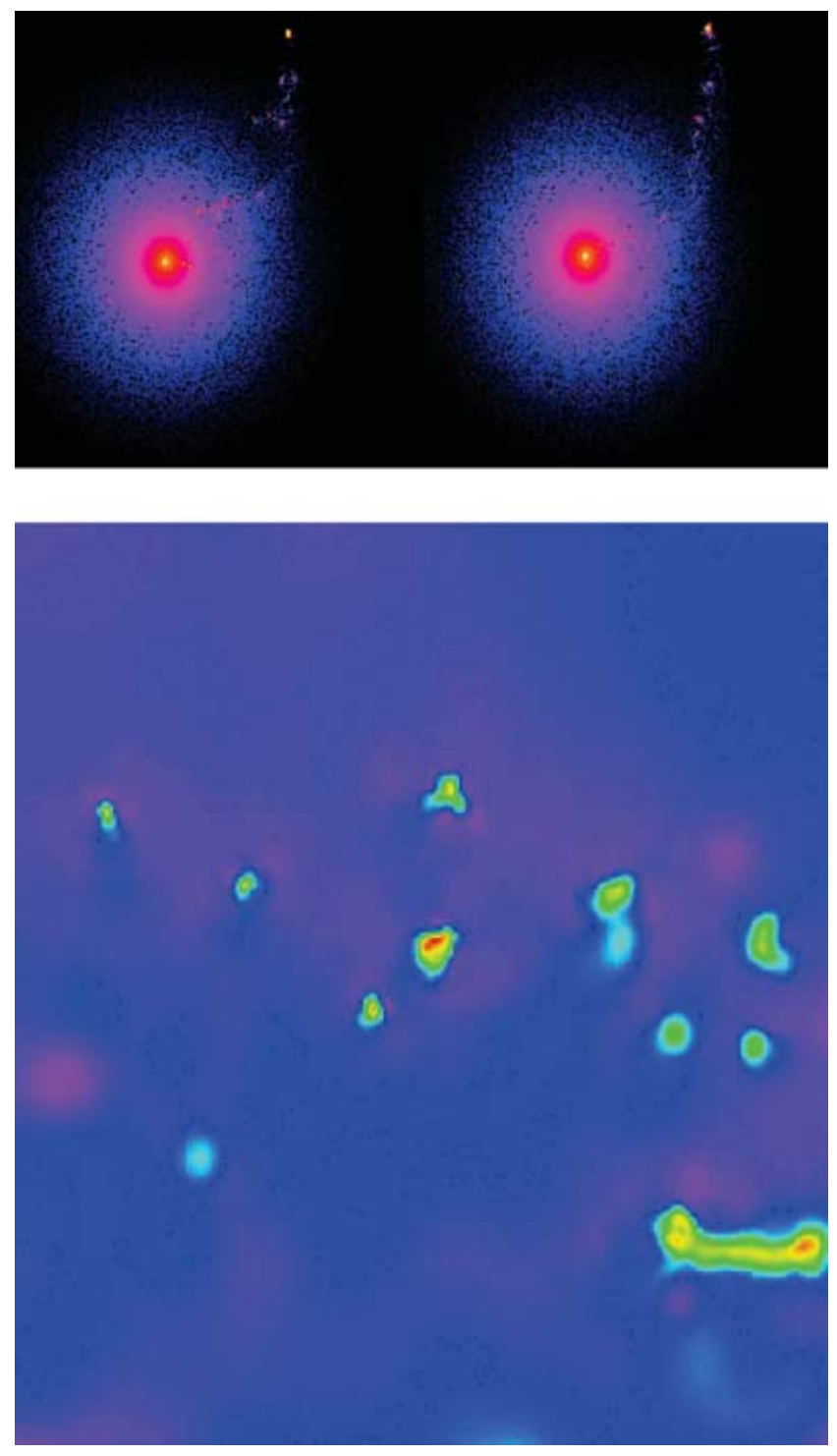

Figure 17. Top: colour-coded density map (brighter colours for higher densities) showing a box of $200 \mathrm{kpc}$ on a side, slightly offset from the centre of mass of the Milky Way+satellite system, for run Rc20RC (left) and run Rc20RCUV (right) at $t=1.5$ Gyr. A gaseous trail fragmenting into clouds is visible. Bottom: colour-coded temperature map of one small region of the gaseous stream in run Rc20RC (the box has a size of about $40 \mathrm{kpc}$ on a side) showing the detailed structure of the clouds. The colour map is such that colours go from red to violet/magenta through yellow and green going from lower to higher temperatures.

that it is premature to draw firm conclusions concerning their structure. None the less the simulations strongly suggest a connection with $\mathrm{H}$ I fragments and possibly other gaseous structures observed in the Local Group like some of the high velocity clouds (HVCs) and the Magellanic Stream (see also Mastropietro et al. 2005).

\section{SUMMARY AND CONCLUSIONS}

The combination of ram pressure and tidal forces can produce systems resembling gas poor dwarf spheroidals from disc-like progenitors. Here, we summarize our findings as follows.

(i) Ram pressure alone can remove completely the gas only in dwarfs having haloes with $V_{\text {peak }} \lesssim 30 \mathrm{~km} \mathrm{~s}^{-1}$. This is consistent with the results found by Marcolini et al. (2003) using different initial conditions and a different numerical technique. The results of the WT runs also compare well with those of Roediger \& Hensler (2005) which were obtained for larger galaxies being subject to ram pressure forces appropriate for low-density environments as those considered here.

(ii) Compressional heating arising as the dwarf travels through the outer medium can significantly enhance ram pressure stripping by producing a more extended gas distribution. As a result, stripping is more effective in adiabatic runs than in cooling runs since in the latter such heating is dissipated.

(iii) The combination of tides and ram pressure is in general more effective at removing gas from the dwarfs than ram pressure alone and always significantly more effective than tides alone. Tidal stripping lowers $V_{\text {peak }}$ and thus the depth of the potential well of the dwarf.

(iv) Tidally induced bar formation opposes ram pressure stripping by driving a large fraction of gas towards the centre, where it is harder to strip at subsequent pericenter passages. This counteracting effect is stronger when radiative cooling is included and explains why in a few cases the addition of tides does not increase the amount of stripped mass.

(v) Low baryonic contents and high $\mathrm{M} / \mathrm{L}$ comparable to those of many dSphs can be obtained as a result of ram pressure and tidal stripping. However, heating sources, such as the cosmic UV background arising during reionization, are required in order to obtain a very low gas fraction in a halo as massive as that in which Draco is probably embedded.

(vi) The gaseous stream produced by the stripped dwarfs fragments into several cold clouds pressure confined by the outer medium. These clouds have properties reminiscent of gaseous structures possibly associated with the Local Group, including some of the HVCs and the Hi fragments recently detected around M31.

We have considered only two types of orbits in this paper. Since proper motions of dSphs are poorly constrained [recent Hubble Space Telescope (HST) based measurement still have a quite large error, e.g. see Piatek et al. 2003 for Carina], there is still quite some freedom in choosing the initial orbits. For example, evolving model V40c20 on an orbit with smaller pericenter, say between 10 and $20 \mathrm{kpc}$, would enhance both tidal stripping and ram pressure. More eccentric orbits would lead to higher pericentric velocities which greatly increase the efficiency of ram pressure stripping. Furthermore, the progenitors of dSphs might have suffered both tidal and ram pressure stripping already before entering the Milky Way halo (Kravtsov et al. 2004). Hence, our results should be regarded as quite conservative.

As we noted above, the initial orbits and initial masses of the dwarfs are both factors that determine the final dark-to-baryonic mass ratio and other properties of the remnants. Therefore, the fact that Fornax and Draco have similar masses, as inferred from their velocity dispersions (Kazantzidis et al. 2004), but differ by more than a factor of 10 in their luminosity, can be explained in two ways. Either the progenitors of these two dwarfs started out with very different relative amounts of dark matter and baryons, for reasons related to their formation history and not to the environment, or they descend from very similar progenitors having comparable dark and baryonic masses, but evolved differently because they entered the primary halo at a different epoch and on different orbits.

The ionizing radiation at high redshift also plays a role in determining the efficiency of ram pressure stripping, and its effect again will be stronger for dwarfs infalling at higher redshift. It is a natural 
consequence of structure formation in hierarchical models that subhaloes that are found closer to the centre of the primary system at $z=0$ are typically those that fell earlier into the potential well of the primary system. Based on their current distances, one would conclude, for example, that Fornax is likely a more recent addition to the Milky Way system, and would have suffered fewer and weaker pericenter passages, relative to Draco. It retained some gas after the first one or two orbits, a condition usually satisfied in those of our simulations that have the largest pericenter, and underwent a few bursts over the past few Gyr (Mayer et al. 2001b). Instead, Draco entered earlier on a tighter orbit and lost almost all its gas at the first pericenter passage, thus ending star formation early. We note that for the orbits with a pericenter of $30 \mathrm{kpc}$, pericenter crossing occurs $0.7 \mathrm{Gyr}$ after the dwarf enters the Milky Way halo. If the dwarf begins its infall at $z=6-7$, i.e. $\sim 1$ Gyr after the big bang, this leaves about $\sim 2$ Gyr to form stars before gas removal occurs, consistent with the estimated time span for the star formation history of Draco (Grebel \& Gallagher 2004). While the infall time of the progenitor of Draco is clearly a free parameter, our results show that these simple assumptions produce a scenario that fits a number of observational constraints.

One caveat in interpreting the results of the simulations as we have just done stems from the fact that we do not include radiative transfer and therefore we cannot estimate directly the effect of selfshielding on the ionization state and cooling of the gas in the dwarfs. If some fraction of the gas is able to self-shield from the UV photons, then it could recombine and cool to the much lower temperatures dictated by molecular cooling, thus being much harder to strip.

Recently, Susa \& Umemura (2004) have performed cosmological simulations with SPH hydrodynamics to study the formation of dwarf galaxies at high redshift, before and after reionization. They include also radiative transfer of the UV photons produced by a cosmic ionizing background with intensity comparable to the one employed by us and find that gas self-shields efficiently only for densities $\rho>10^{-22} \mathrm{~g} \mathrm{~cm}^{-3}$. In both the FI runs and WT runs, with models V28c4 or V40c20, the gas has always densities more than an order of magnitude lower than this threshold; even during the phase in which the density increases the most, namely after the bar-driven gas inflow, we measure $\rho \sim 3 \times \sim 10^{-24} \mathrm{~g} \mathrm{~cm}^{-3}$. This is because the initial gas distribution in our model galaxies has by construction a very low characteristic density (as seen in dIrr galaxies) which both the compression exerted by the low-density gaseous background and the bar instability cannot increase enough. Instead, in cooling runs with model V60c4 the density becomes higher than the characteristic threshold for self-shielding identified by Susa \& Umemura (2004) as a result of the bar-driven inflow, $\rho \sim 5 \times 10^{-22} \mathrm{~g} \mathrm{~cm}^{-3}$, while it is always lower than such a threshold for all the gas that does not partake of the inflow. Since the latter is essentially all the gas that is stripped this implies that self-shielding should not affect the estimate of the stripped mass even in these runs.

We note that if the gas is not able to self-shield from the UV photons, then it will not be able to form a molecular phase and cool further to the low temperatures necessary for molecular clouds to form and star formation to begin. This confirms that no star formation should occur in most of our models during the phase in which the UV background is at its peak, namely before $z=2$. It also shows the fact that we miss low-temperature coolants, so that gas can never cool radiatively below $10^{4} \mathrm{~K}$, is not an issue for the main results of this paper. Instead, molecular cooling and star formation should be included to study in detail the evolution of the only model (V60c4) that appears to be able to maintain a neutral gas fraction and possibly form stars even in the presence of the cosmic UV background.
It is important to keep in mind that the Milky Way halo model used here is based on data at $z=0$ and the components that we assumed as fixed do indeed evolve through cosmic time. At high $z$, as the dwarfs began their route towards the Milky Way, the dark halo and gaseous halo masses may have been quite different. However, $\Lambda \mathrm{CDM}$ galaxy formation simulations suggest that large disc galaxies had the last major merger quite early on in order for a large enough disc to assemble by $z=0$, which means most of the dark halo mass was probably already in place at $z=2-3$. The evolution of the gaseous halo in $\Lambda \mathrm{CDM}$ simulations has not been investigated yet, but work in progress suggests its density at several tens of kpc could have been 10 times higher than the value adopted in this paper. In this paper, we have considered only one particular value of the baryon fraction/disc mass fraction. Models with the same spin parameter and a lower disc mass fraction would develop weaker tidally triggered stellar bars, producing much weaker gas inflows and therefore enhancing the effect of ram pressure stripping. The same result is achieved by reducing the stellar fraction at fixed disc mass fraction as we have briefly reported in Section 3.3 (Mayer et al., in preparation). This type of systems might indeed be the best candidate as progenitors of the faintest among dSphs. Stronger inflows and thus less potential for stripping are instead expected for dwarfs with more massive baryonic components. The latter would resemble the high surface brightness (HSB) dwarf models studied in Mayer et al. (2001b); according to the results of that work these galaxies would undergo a very intense star formation owing to tidal triggering near pericenter, producing a HSB central stellar core as that of the bright $\mathrm{dE}$ satellites of M31 (e.g. NGC 205).

In our simulations, ram pressure stripping is most effective close to pericentric passages. This reflects the fact that we have assumed a smooth density distribution for the hot halo. If the halo has a complex multiphase structure as predicted by some models (Maller \& Bullock 2004), the dwarfs would move through a clumpy medium and could undergo intense ram pressure stripping even far from pericenter if they encounter a dense and massive cloud. Galaxy formation simulations which follow the build-up and evolution of the gaseous halo at very high resolution with a proper treatment of the multiphase ISM will eventually be able to address this aspect. However, current cosmological simulations can hardly capture the effect of ram pressure on satellite galaxies even in the case of a smooth halo because of the limited resolution and owing to uncertainties in the star formation and feedback processes which concur to determine the gas fraction in satellite galaxies. Therefore, nowadays simulations, such as those discussed in this paper, represent a necessary counterpart to cosmological runs and provide the only means to test the physical effect of environmental mechanisms in a robust way.

\section{ACKNOWLEDGMENTS}

We thank the anonymous referee for several helpful comments and suggestions. The numerical simulations were carried out on the zBox supercomputer at the University of Zurich and on LeMieux at the Pittsburgh Supercomputing Centre.

\section{REFERENCES}

Abadi M. G., Moore B., Bower R. G., 1999, MNRAS, 308, 947

Balsara D. S., 1995, J. Comput. Phys., 121, 357

Barkana R., Loeb A., 1999, ApJ, 523, 54

Benson A. J., Lacey C. G., Baugh C. M., Cole S., Frenk C. S., 2002a, MNRAS, 333, 156B 
Benson A. J., Frenk C. S., Lacey C. G., Baugh C. M., Cole S., 2002b, MNRAS, 333, 177

Blitz L., Robishaw T., 2000, ApJ, 541, 675

Braun R., Thilker D. A., 2004, A\&A, 417, 421

Bullock J. S., Kravtsov A. V., Weinberg D. H., 2000, ApJ, 539, 517

Bullock J. S., Kolatt T. S., Sigad Y., Somerville R. S., Kravtsov A. V., Klypin A. A., Primack J. R., Dekel A., 2001, MNRAS, 321, 559

Cowie L. L., McKee C. F., 1977, ApJ, 211, 135

de Blok W. J. G., McGaugh S. S., 1997, MNRAS, 290, 533

Dehnen W., Binney J., 1998, MNRAS, 294, 429

Dekel A., Silk J., 1986, ApJ, 303, 39

Einasto J., Saar E., Kaasik A., Chemin A. D., 1974, Nat, 252, 111

Faber S. M., Lin D. C. N., 1983, ApJ, 266, L17

Farouki R., Shapiro S. L., 1980, ApJ, 241, 928

Ferguson H. C., Binggeli B., 1994, A\&AR, 6, 57

Ferrara A., Tolstoy E., 2000, MNRAS, 313, 291

Gallagher J. S., Madsen G. J., Reynolds R. J., Grebel E. K., Smecker-Hane T. A., 2003, ApJ, 588, 326

Gallart C., Freedman W. L., Aparicio A., Betelli G., Chiosi C., 1999, AJ, 118,2245

Gallart C., Martinez-Delgad0 D., Gomez-Flechoso M. A., Mateo M., 2001, AJ, 121,2572

Gardner J. P., 2001, ApJ, 557, 616

Gavazzi G., Boselli A., Mayer L., Iglesias-Paramo J., Vilchez J. M., Carrasco L., 2001, ApJ, 563, L23

Ghigna S., Moore B., Governato F., Lake G., Quinn T., Stadel J., 1998, MNRAS, 300, 146

Gill S. P. D., Knebe A., Gibson B., 2004, MNRAS, 351, 410

Governato F., Mayer L., Wadsley J., Gardner J. P., Willman B., Hayashi E., Quinn T., Stadel J., Lake G., 2004, ApJ, 607, 688

Grebel E. K., Gallagher J. S., 2004, ApJ, 610, L89

Grebel E. K., Gallagher J. S., Herbeck D., 2003, ApJ, 125, 1926

Guhathakurta P., Reitzel D. B., 1998, in Zaritsky D., ed., ASP Conf. Ser. Vol. 136, Galactic Halos. Astron. Soc. Pac., San Francisco, p. 22

Gunn J. E., Gott J. R. I., 1972, ApJ, 176, 1

Haardt F., Madau P., 1996, ApJ, 461, 20

Hernandez X., Gilmore G., Valls-Gabaud D., 2000, MNRAS, 317, 831

Hernquist L., 1993, ApJS, 86, 389

Hogan C. J., Dalcanton J. J., 2000, Phys. Rev. D, 62, 063511

Jimenez R., Verde L., Oh S. P., 2003, MNRAS, 339, 243

Kazantzidis S., Mayer L., Mastropietro C., Diemand J., Stadel J., Moore B., 2004, ApJ, 608, 663

Kleyna J., Wilkinson M. I., Evans N. W., Gilmore G., Frayn C., 2002, MNRAS, 330, 792

Kleyna J., Wilkinson M. I., Evans N. W., Gilmore G., 2005, ApJ, 630, L141

Klypin A., Zhao H., Somerville R. S., 2002, ApJ, 573, 597

Kravtsov A. V., Gnedin O. Y., Klypin A. A., 2004, ApJ, 609, 482

Lin D. N. C., Jones B. F., Klemola A. R., 1995, ApJ, 439, 652

Lokas E. L., 2002, MNRAS, 333, 697

Lufkin G., Quinn T., Wadsley J., Stadel J., Governato F., 2004, MNRAS, 347,421

Majewski S. R., Ostheimer J. C., Kunkel W. E., Johnston K. V., Patterson R. J., Palma C., 1999, in Chu Y. H., Suntzeff N. B., Hesser J. E., Bohlender D. A., eds, IAU Symp. Vol. 190, New Views of the Magellanic Clouds. Astron. Soc. Pac., San Francisco, p. 508

Maller A. H., Bullock J. S., 2004, MNRAS, 355, 694

Marcolini A., Brighenti F., D'Ercole A., 2003, MNRAS, 345, 1329

Marcolini A., Brighenti F., D’Ercole A., 2004, MNRAS, 352, 363

Mashchenko S., Carignan C., Bouchard A., 2004, MNRAS, 352, 168

Mastropietro C., Moore B., Mayer L., Wadsley J., Stadel J., 2005, MNRAS, 363,509

Mateo M., 1998, ARA\&A, 36, 435
Mathewson D. S., Ford V. L., Schwarz M. P., Murray J. D., 1979, IAU Symp. Vol. 84, The Large-Scale Characteristics of the Galaxy. Reidel, Dordrecht, p. 547

Mathewson D. S., Wayte S. R., Ford V. L., Ruan K., 1987, Proc. Astron. Soc. Aust., 7, 19

Mayer L., 2004, in Dettmar R., Klein U., Salucci P., Proc. Baryons in Dark Matter Halos. SISSA, in press (astro-ph/0411476)

Mayer L., Moore B., 2004, MNRAS, 354, 477

Mayer L., Wadsley J., 2004a, in Prada F., Martinez-Delgado D., Mahoney T., eds, ASP Conf. Ser. Vol. 327, Satellites and Tidal Streams. Astron. Soc. Pac., San Francisco p. 230

Mayer L., Wadsley J., 2004b, MNRAS, 347, 277

Mayer L., Governato F., Colpi M., Moore B., Quinn T., Wadsley J., Stadel J., Lake G., 2001a, ApJ, 547, L123

Mayer L., Governato F., Colpi M., Moore B., Quinn T., Wadsley J., Stadel J., Lake G., 2001b, 559, 754

Mayer L., Moore B., Quinn T., Governato F., Stadel J., 2002, MNRAS, 336, 119

Mo H. J., Mao S., White S. D. M., 1998, MNRAS, 295, 319

Moore B., Katz N., Lake G., 1996, ApJ, 457, 455

Mori M., Burkert A., 2000, ApJ, 538, 559

Mori M., Ferrara A., Madau P., 2002, ApJ, 571, 40

Murray S. D., White S. D. M., Blondin J. M., Lin D. N. C., 1993, ApJ, 407, 588

Navarro J. F., Frenk C. S., White S. D. M., 1996, ApJ, 462, 563

Nulsen P. E. J., 1982, MNRAS, 198, 1007

Odenkirchen M. et al., 2001, AJ, 122, 2538

Piatek S. et al., 2003, AJ, 126, 2346

Quilis V., Moore B., 2001, ApJ, 555, L95

Quilis V., Moore B., Bower R., 2000, Sci, 288, 1617

Read J. I., Gilmore G., 2004, MNRAS, 356, 107

Roediger E., Hensler G., 2005, A\&A, 433, 875

Schulz S., Struck C., 2001, MNRAS, 328, 185

Sembach K. R. et al., 2003, ApJS, 146, 165

Shaviv N. J., Dekel A., 2003, MNRAS, submitted (astro-ph/0305527)

Sommer-Larsen J., Gotz M., Portinari L., 2003, ApJ, 596, 47

Somerville R. S., 2002, ApJ, 572, L23

Springel V., Hernquist L., 2003, MNRAS, 339, 289

Springel V., White S. D. M., 1999, MNRAS, 307, 162

Susa H., Umemura M., 2004, ApJ, 600, 1

Taffoni G., Mayer L., Colpi M., Governato F., 2003, MNRAS, 341, 434

Thacker R. J., Couchman H. M. P., 2000, ApJ, 545, 728

Thilker D. A., Braun R., Walterbros A. M., Corbelli E., Lockman F. J., Murphy E., Maddalena R., 2004, ApJ, 601, L39

Thoul A. A., Weinberg D. H., 1996. ApJ, 466, 608

Tittley E. R., Pearce F. R., Couchman H. M. P., 2001, ApJ, 561, 69

Toyama K., Ikeuchi S., 1980, Prog. Theor. Phys., 64, 831

Van den Bergh S., 1996, ApJ, 428, 617

Verde L., Oh S. P., Jimenez R., 2002, MNRAS, 336, 541

Vietri M., Ferrara A., Miniati F., 1997, ApJ, 483, 262

Vollmer B., 2003, A\&A, 398, 525

Vollmer B., Cayatte V., Boselli A., Balkowski C., Duschl W. J., 1999, A\&A, 349,411

Vollmer B., Marcelin M., Amram P., Balkowski C., Cayatte V., Garrido O., 2000, A\&A, 364, 532

Vollmer B., Braine J., Balkowski C., Cayatte V., Duschl W. J., 2001, A\&A, 374,824

Wadsley J. W., Stadel J., Quinn T., 2004, New Astron., 9, 137

White S. D. M., Frenk C. S., 1991, ApJ, 379, 52

Wilkinson M., Kleyna J. T., Evans W. N., Gilmore G. F., Irwin M. J., Grebel E. K., 2004, ApJ, 611, L21

This paper has been typeset from a $\mathrm{T}_{\mathrm{E}} \mathrm{X} / \mathrm{LT}_{\mathrm{E}} \mathrm{X}$ file prepared by the author. 